ARTICLE

https://doi.org/10.1038/s41467-021-24407-y

\title{
M1BP cooperates with CP190 to activate transcription at TAD borders and promote chromatin insulator activity
}

\author{
Indira Bag (1) 1,2, Shue Chen (1) 1,2,4, Leah F. Rosin (1) 1,2,4, Yang Chen (1) 1,2, Chen-Yu Liu ${ }^{3}$, Guo-Yun Yu ${ }^{3}$ \& \\ Elissa P. Lei (10 ${ }^{1,2 \otimes}$
}

Genome organization is driven by forces affecting transcriptional state, but the relationship between transcription and genome architecture remains unclear. Here, we identified the Drosophila transcription factor Motif 1 Binding Protein (M1BP) in physical association with the gypsy chromatin insulator core complex, including the universal insulator protein CP190. M1BP is required for enhancer-blocking and barrier activities of the gypsy insulator as well as its proper nuclear localization. Genome-wide, M1BP specifically colocalizes with CP190 at Motif 1-containing promoters, which are enriched at topologically associating domain (TAD) borders. M1BP facilitates CP190 chromatin binding at many shared sites and vice versa. Both factors promote Motif 1-dependent gene expression and transcription near TAD borders genome-wide. Finally, loss of M1BP reduces chromatin accessibility and increases both interand intra-TAD local genome compaction. Our results reveal physical and functional interaction between CP190 and M1BP to activate transcription at TAD borders and mediate chromatin insulator-dependent genome organization.

\footnotetext{
${ }^{1}$ Nuclear Organization and Gene Expression Section, Bethesda, MD, USA. ${ }^{2}$ Laboratory of Biochemistry and Genetics, Bethesda, MD, USA. ${ }^{3}$ Laboratory of Cellular and Developmental Biology, National Institute of Diabetes and Digestive and Kidney Diseases, National Institutes of Health, Bethesda, MD, USA.

${ }^{4}$ These authors contributed equally: Shue Chen, Leah F. Rosin. ${ }^{凶}$ email: leielissa@niddk.nih.gov
} 
n eukaryotic cells, the three-dimensional organization of the genome plays a critical role in achieving proper spatial and temporal patterns of gene expression during development. Chromatin insulators are DNA-protein complexes involved in the establishment, maintenance, and regulation of nuclear organization to modulate gene expression (reviewed in ${ }^{1,2}$ ). Insulators regulate interactions between cis-regulatory elements such as enhancers and promoters and demarcate silent and active chromatin regions to ensure their proper regulation. They can inhibit the interaction between an enhancer and a promoter when positioned between the two elements and can act as a barrier to stop repressive chromatin from spreading over active genes. Furthermore, chromatin insulators can promote intra- and interchromosomal looping to control topology of the genome. Certain insulator proteins are highly enriched at the self-interacting boundaries of topologically associating domains (TADs) throughout the genome. In mammals, only a single insulator protein, CCCTC-binding Factor (CTCF), has thus far been identified, and CTCF indeed is enriched at TAD borders and is required for TAD formation. In contrast, Drosophila melanogaster CTCF is not particularly enriched at TAD borders, and a recent study indicates that CTCF plays a limited role in TAD formation in flies ${ }^{3}$. In fact, Drosophila harbors a variety of insulator protein complexes, all of which contain the protein Centrosomal protein 190 (CP190). CP190 is highly enriched at TAD borders, suggesting a possible role in TAD formation. Another notable feature of genome organization that has been explored in detail in Drosophila is the key role of transcription and the presence of constitutively active genes at TAD borders ${ }^{4-6}$. General inhibition of transcription using chemical treatments or heat shock results in disruption of TADs and compartments, but the mechanistic details of how transcription contributes to genome organization are yet to be elucidated.

The Drosophila gypsy insulator, also known as the Suppressor of Hairy wing $[\mathrm{Su}(\mathrm{Hw})]$ insulator, was the first characterized CP190containing insulator complex. The zinc-finger DNA-binding protein $\mathrm{Su}(\mathrm{Hw})$ provides binding specificity of the complex, and both $\mathrm{CP} 190$ and the Modifier of $\operatorname{mdg} 4[\operatorname{Mod}(\operatorname{mdg} 4)] 67.2 \mathrm{kDa}$ isoform [Mod(mdg4)67.2] contain an N-terminal Broad-Complex, Tramtrack, and Bric a brac (BTB) domain that can homodimerize or heterodimerize to facilitate insulator-insulator interactions and promote formation of long range insulatormediated loops ${ }^{7-10}$. Initially, the gypsy insulator complex was characterized as binding the 5'-untranslated region of the gypsy retroelement. However, the core complex also binds thousands of endogenous sites throughout the genome and can function similarly at least at a subset of those sites ${ }^{11-15}$. Moreover, the three gypsy insulator core components do not colocalize absolutely at all binding sites throughout the genome, and each protein can interact with other insulator proteins ${ }^{13,16-19}$. In diploid interphase nuclei, gypsy insulator proteins coalesce into large foci termed insulator bodies. These structures can be induced by stress ${ }^{20}$, and insulator bodies have also been proposed to serve as storage depots for insulator proteins ${ }^{21,22}$. Nevertheless, there is a high correlation between proper insulator function and insulator body localization $7,10-16,23-27$. In summary, the gypsy insulator complex contributes to higher order nuclear organization on several levels.

CP190 also associates with a variety of additional DNAbinding proteins that likely impart specificity of the respective complex. The BED finger-containing proteins BEAF-32, Ibf1, and Ibf2 interact with CP190 and promote insulator function ${ }^{17}$. Three additional zinc-finger proteins Pita, ZIPIC, and CTCF also interact with CP190 and contribute to insulator activity ${ }^{18,28}$. Recently, the zinc-finger protein CLAMP was demonstrated to positively affect gypsy insulator activity and to colocalize particularly with CP190 at promoters throughout the genome ${ }^{16}$. In addition, previous work showed that CP190 preferentially binds Motif 1-containing promoters ${ }^{29}$, but the functional significance of this observation is currently unknown. The precise functions of CP190, its associated factors, as well as their relationship with transcription regulation have not yet been elucidated.

Motif 1 binding protein (M1BP) is a ubiquitously expressed transcriptional activator that is required for the expression of predominantly constitutive genes. A zinc-finger DNA-binding protein, M1BP, specifically binds to the core promoter element Motif 1 consensus sequence that is distinct from the canonical TATA box and mainly controls the expression of constitutively active genes that are transiently paused ${ }^{30}$. For example, M1BP interacts with the TATA-binding protein-related factor 2 (TRF2) to activate transcription of ribosomal protein genes in a Motif 1dependent manner ${ }^{31}$. Finally, recent studies found that Motif 1 and M1BP are highly enriched at TAD boundaries along with CP190 and BEAF-32 $32-35$. Depletion of M1BP led to increased inter-chromosomal Hi-C contacts; however, concomitant cell cycle disruption precluded interpretation of these results ${ }^{33}$. The possible role of M1BP-dependent transcriptional regulation in genome organization has not yet been interrogated in detail.

In this study, we identify M1BP as a physical interactor and positive regulator of the gypsy insulator complex. Depletion of M1BP decreases gypsy-dependent enhancer blocking and barrier activities and reduces the association of the core insulator complex with the gypsy insulator sequence. ChIP-seq analysis reveals extensive genome-wide overlap of M1BP particularly with promoter-bound CP190, and depletion of M1BP results in extensive loss of CP190 chromatin association genome-wide. Depletion of CP190 also disrupts M1BP binding at many of its binding sites. Nascent euRNA-seq (neuRNA-seq) analysis of M1BP- or CP190-depleted cells indicates that both factors coregulate a similar set of genes genome-wide. In particular, loss of gene activation correlates with disrupted M1BP and CP190 binding, and these events are frequently observed at TAD borders. Depletion of M1BP disrupts gypsy insulator body localization within the nucleus and alters both inter- and intra-TAD local genome compaction. Finally, knockdown of M1BP decreases chromatin accessibility at its binding sites, including genes that it activates and regions in proximity of TAD borders. Taken together, our findings identify a mechanistic relationship between M1BP and CP190 to activate Motif 1-dependent transcription as well as to promote chromatin insulator activity and nuclear organization.

\section{Results}

M1BP interacts physically with core gypsy insulator proteins. In order to identify additional gypsy insulator interactors, we performed immunoaffinity purification of Drosophila embryonic nuclear extracts using antibodies specific for $\mathrm{Su}(\mathrm{Hw})$ or CP190 and analyzed the eluates using quantitative mass spectrometry. In addition to the third gypsy core component $\operatorname{Mod}(\operatorname{mdg} 4) 67.2$ and previously reported interactors such as Ibf1, Ibf2, BEAF-32, and CTCF, we identified M1BP in both purifications (Supplementary Tables 1 and 2). CP190 was also previously identified as a physical interactor by co-immunoprecipitation of M1BP by mass-spec analysis using Drosophila embryonic nuclear extract and subsequently confirmed by western blotting ${ }^{36}$. We verified that all three gypsy core components interact with M1BP by performing anti-Su(Hw), anti-CP190, and anti-Mod(mdg4)67.2 purifications and western blotting (Fig. 1a-c). Finally, we verified that $\mathrm{Su}(\mathrm{Hw})$, CP190, and Mod(mdg4)67.2 could be immunopurified by antiM1BP but not normal serum (Fig. 1d). In contrast, an unrelated factor, Polycomb, was not co-immunoprecipitated with any of these antibodies. These results indicate that M1BP is associated 
a

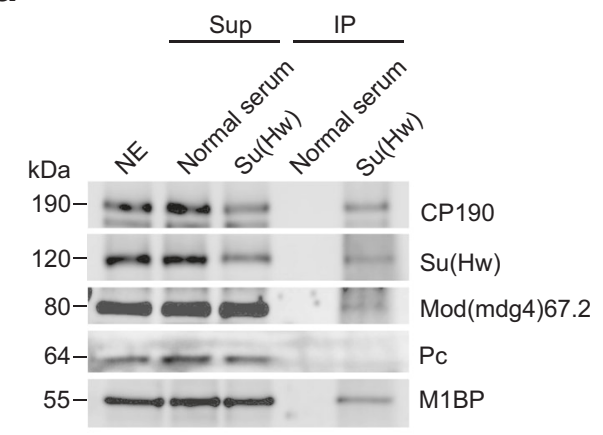

C

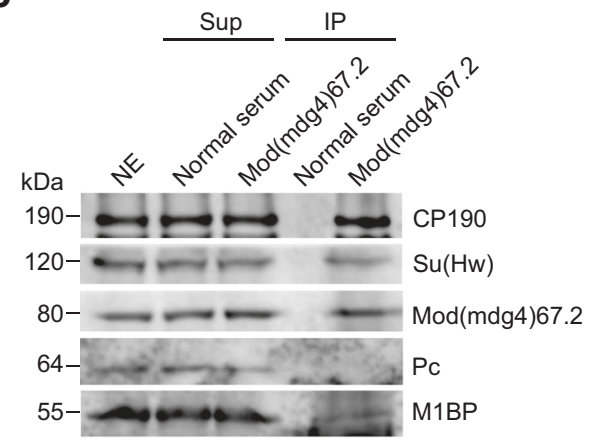

b

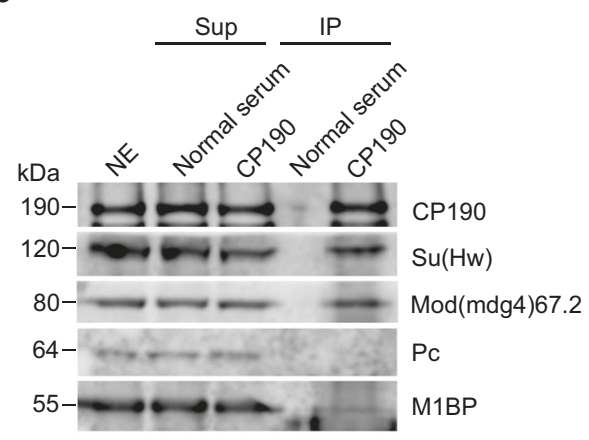

d

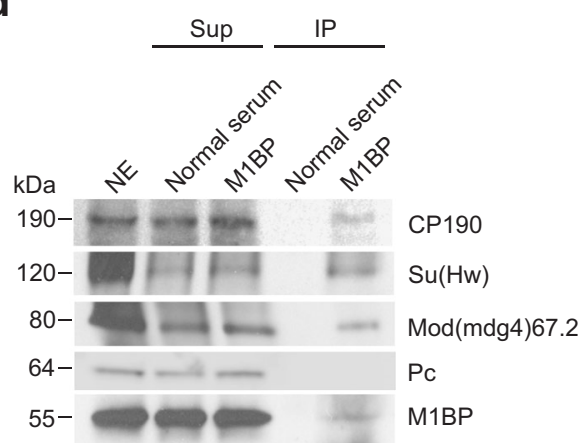

Fig. 1 Co-immunoprecipitation of core gypsy components with M1BP. a Nuclear extracts (NE) from embryos aged 0-24 h were immunoprecipitated with either normal guinea pig serum or guinea pig anti-Su(Hw) antibody. Unbound supernatant (Sup) and bound (IP) fractions are shown. Polycomb (Pc) is shown as a negative control. In all, $10 \%$ of NE is loaded in the gel. We calculated that $5.2 \%$ of total CP190, 7.2\% of Su(Hw), 1.9\% of Mod(mdg4)67.2, and $5.3 \%$ of M1BP were immunoprecipitated with anti-Su(Hw) antibody. b Immunoprecipitation of M1BP with normal guinea pig serum or guinea pig antiCP190 is shown. Overall, $12 \%$ of total CP190, 7.0\% of Su(Hw), 8.2\% of Mod(mdg4)67.2, and 3.8\% of M1BP were immunoprecipitated with anti-CP190 antibody. c Immunoprecipitation of M1BP with normal rabbit serum or rabbit anti-Mod(mdg4)67.2 is shown. Overall, $11 \%$ of total CP190, $8.5 \%$ of Su(Hw), $10 \%$ of Mod(mdg4)67.2, and 3.0\% of M1BP were immunoprecipitated with anti-Mod(mdg4)67.2 antibody. d Immunoprecipitation of M1BP with normal rabbit serum or rabbit anti-M1BP is shown. Overall, 3.8\% of total CP190, 2.6\% of Su(Hw), 1.4\% of Mod(mdg4)67.2, and 3.7\% of M1BP were immunoprecipitated with anti-M1BP antibody. Samples from the same experiment were run on different gels for proteins with similar molecular weights. All western blotting experiments were performed with at least two independent biological replicates for each antibody, and a single experiment is shown.

physically either directly or indirectly with the core gypsy insulator proteins.

M1BP promotes gypsy insulator function. Given their physical interaction, we sought to test whether M1BP may play a role in gypsy insulator function. To deplete M1BP levels in vivo, we utilized an $M 1 B P^{R N A i}$ fly line expressing a hairpin under upstream activating sequence (UAS) control that generates siRNAs against $M 1 B P$ when combined with a Gal4 driver. To validate the knockdown efficiency of this line, we performed western blot analysis of larval extracts from flies expressing this RNAi construct using the ubiquitously expressed Act5C-Gal4 driver compared to a control line expressing driver alone (Fig. 2a). Importantly, depletion of M1BP does not have any effect on overall $\mathrm{Su}(\mathrm{Hw}), \mathrm{CP} 190$ or $\operatorname{Mod}(\operatorname{mdg} 4) 67.2$ protein levels. Using Act5C-Gal4, M1BPRNAi resulted in $100 \%$ late larval lethality, and complete pupal lethality is observed using the muscle-specific Mef2-Gal4 driver (Supplementary Table 3). With CNS-enriched $l$ (3)31-1-Gal4 or wing-expressed Ser-Gal4, M1BPRNAi expression, flies remain completely viable.

In order to investigate whether M1BP affects gypsy-dependent enhancer-blocking activity, we examined the effect of M1BP depletion on the well-characterized allele $c t^{6}$. This loss-offunction allele results from gypsy retrotransposon insertion between the promoter and distal wing margin enhancer of cut (Fig. 2b). The gypsy insulator blocks communication between the two elements, reducing cut expression and causing disruption of the wing margin visible in the adult fly ${ }^{37}$. We used a scoring scale from 0 to 4 with increasing severity of wing margin notching corresponding to higher insulator activity. We found that knockdown of M1BP driven by Ser-Gal4 compared to driver alone restored wing margin tissue, consistent with a decrease in enhancer-blocking activity (Fig. 2c). We also determined that knockdown of $M 1 B P$ does not result in changes in wing margin induced by the gypsy-independent $c t^{n}$ loss-of-function allele, which is caused by insertion of the roo transposable element (Supplementary Fig. 1). These results indicate that M1BP is required for gypsy-dependent enhancer blocking activity.

We next investigated the effect of M1BP depletion on gypsydependent barrier activity in a variety of tissues. In this assay, we used a UAS-luciferase reporter that is either insulated by flanking $\mathrm{Su}(\mathrm{Hw})$-binding sites or not insulated, with either reporter inserted into the same genomic site (Fig. 2d) ${ }^{24}$. We performed this quantitative luciferase-based assay using different tissuespecific Gal4 drivers to control both the luciferase reporter and $M 1 B P^{R N A i}$. Ubiquitously expressed Act5C-Gal4 promotes high luciferase expression in insulated compared to non-insulated control larvae (Fig. 2e and Supplementary Table 4). As a positive control, knockdown of $s u(H w)$ resulted in dramatic decrease in luciferase expression only in the insulated line, suggesting loss of barrier function. Likewise, knockdown of M1BP driven by Act5CGal4 resulted in statistically significant reduction in luciferase activity compared to the insulated control line. Furthermore, knockdown using either the muscle-specific Mef2-Gal4 driver or the CNS-enriched l(3)31-1-Gal4 driver also resulted in significant decreases in luciferase activity (Fig. 2f, g and Supplementary 
a

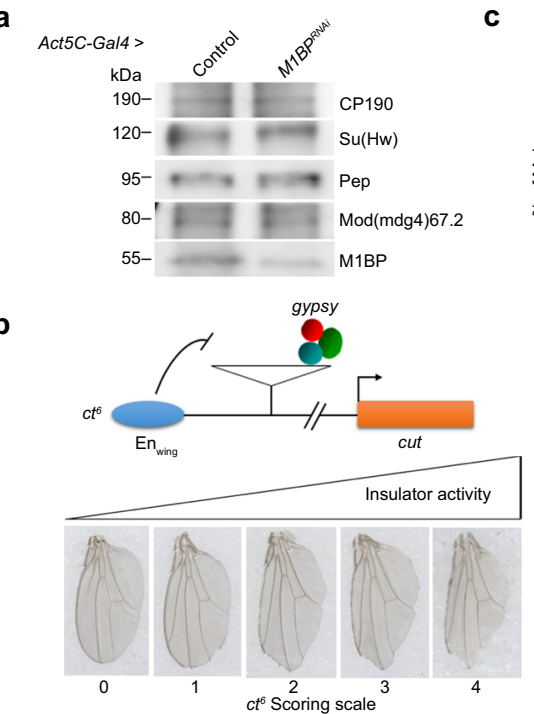

e

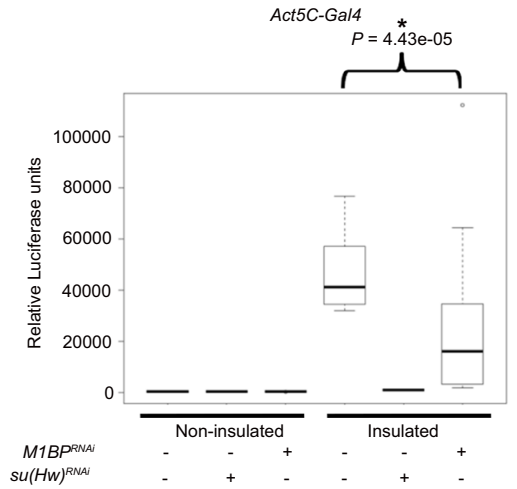

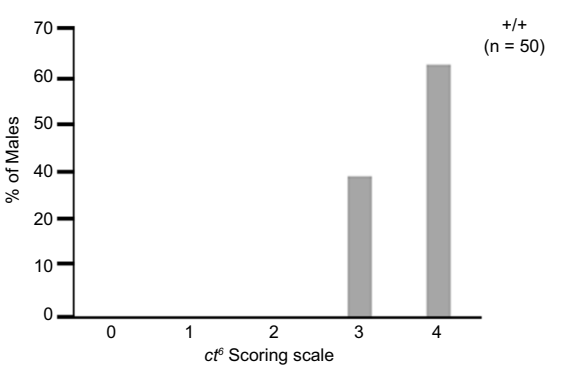

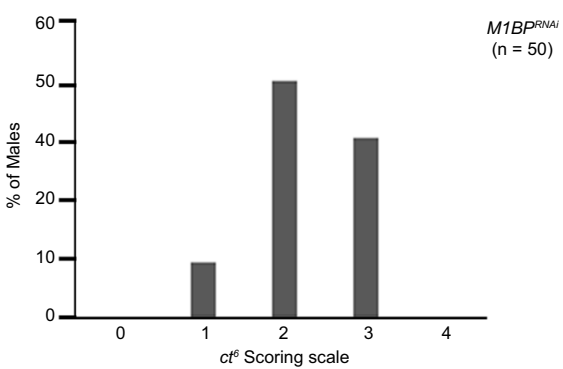

d

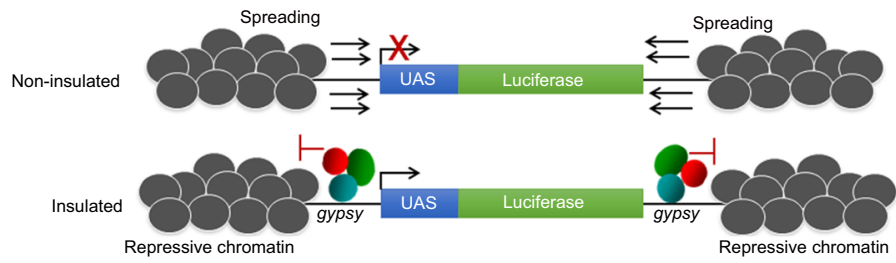

f

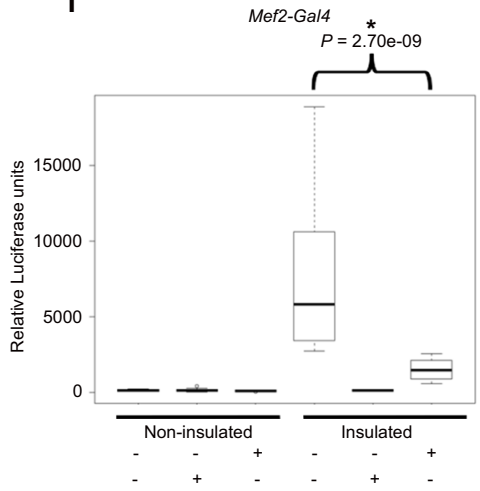

g

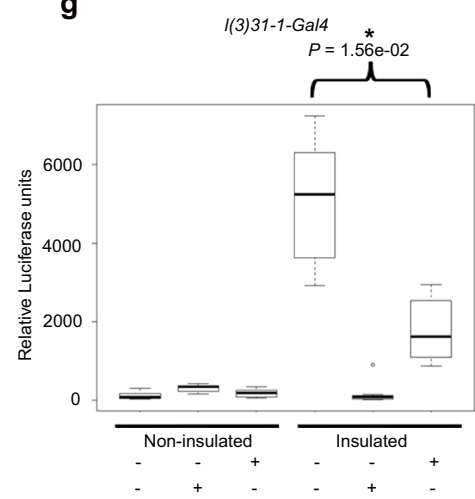

Fig. 2 M1BP promotes gypsy-dependent enhancer-blocking and barrier activities. a Western blotting of male third instar larval extracts for M1BP, insulator proteins, and Pep loading control in control and M1BPRNAi knockdown flies using Act5C-Gal4 driver. Samples from the same experiment were run on different lanes of the same gel for proteins with similar molecular weights. Western blotting experiments were performed using two independent biological replicates with similar results. b M1BP promotes enhancer-blocking activity at $c t^{6}$. Top: schematic diagram of reporter system. The gypsy retrotransposon is inserted in between the promoter of cut and wing margin (En Wing) enhancer. Bottom: insulator activity for ct ${ }^{6}$ was scored in male flies on a scale of $0-4$. 0 , no notching; 1 , slight notching in anterior tip of wing; 2 , mild notching throughout posterior wing; 3, extensive notching both in anterior and posterior wing; 4 , severe notching throughout the anterior and posterior wing. c Graph represents quantification of $c t^{6}$ wing phenotype of male wildtype $(+/+)$ and M1BPRNAi using Ser-Gal4 driver. $n$, total number of flies scored. d Depletion of M1BP shows reduced gypsy-dependent barrier activity in all tissues tested. Schematic diagram of non-insulated UAS-luciferase system shows spreading of repressive chromatin can reduce luciferase expression, but presence of the gypsy insulator acts as a barrier and allows for luciferase activity. e Relative luciferase activity of insulated or non-insulated male larvae of control and M1BPRNAi driven by Act5C-Gal4 driver, $\mathbf{f}$ Mef2-Gal4 driver, and $\mathbf{g}$ 1(3)31-1-Gal4 driver. Luciferase values of all genotypes are plotted as box and whisker plots using one-way ANOVA followed by Tukey HSD post hoc tests to calculate $P$ values for pairwise comparisons. The box represents the 25-75th percentiles, and the median is indicated. The whiskers show the minimum and maximum values. For each genotype, $n=12$ individual larvae. Bracket indicates $P$ values of two-way comparisons $\left({ }^{\star} P<0.05\right)$ and all comparisons are shown in Supplementary Table 4.

Table 4). We conclude that M1BP promotes gypsy-dependent barrier activity in all tissues tested.

M1BP extensively colocalizes with CP190 genome-wide. In order to obtain high-resolution information about the genomewide chromatin association of M1BP and its relationship with gypsy core components, we performed ChIP-seq analysis for M1BP, CP190, Su(Hw), and Mod(mdg4)67.2 in the embryonic Kc167 (Kc) hemocyte cell line. Using previously validated antibodies, we identified $3121 \mathrm{M} 1 \mathrm{BP}, 8022 \mathrm{CP} 190,4638 \mathrm{Su}(\mathrm{Hw})$, and $3536 \operatorname{Mod}(\operatorname{mdg} 4) 67.2$ peaks. By western blot analysis, we validated efficient protein depletion of M1BP and no effect on insulator protein levels 5 days after $M 1 B P$ double stranded RNA (dsRNA) transfection (Fig. 3a and Supplementary Fig. 2a). Similar to previous work ${ }^{30}$, we also verified that this level of
M1BP depletion did not greatly alter cell viability or lead to accumulation of cells in M-phase (Supplementary Fig. 2b, d-e), in contrast to a previous study that used more dsRNA for a longer time period ${ }^{33}$. Our ChIP-seq analyses confirm that depletion of M1BP by RNAi dramatically reduced M1BP binding to chromatin throughout the genome, also confirming the specificity of the antibody (Fig. 3b). Interestingly, we found that $79 \%$ (2461) of total M1BP peaks overlap with CP190 genome-wide (31\% of total CP190 peaks) (Fig. 3b, c). In contrast, we found very low overlap of M1BP with either $\mathrm{Su}(\mathrm{Hw})(5.2 \%)$ or $\operatorname{Mod}(\operatorname{mdg} 4) 67.2(7.0 \%)$ (Fig. 3c). Finally, M1BP also overlaps considerably with BEAF-32 (41\%) but less substantially with CLAMP, ZIPIC, Pita, Ibf1, Ibf2, and CTCF (Supplementary Fig. 3).

We further examined the distribution of M1BP, CP190, and shared M1BP-CP190 sites with respect to genomic features. Consistent with earlier reports, we verified that both M1BP and 
a

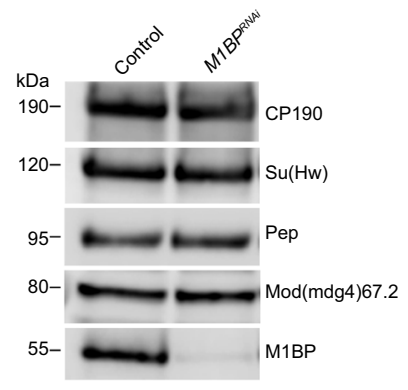

C

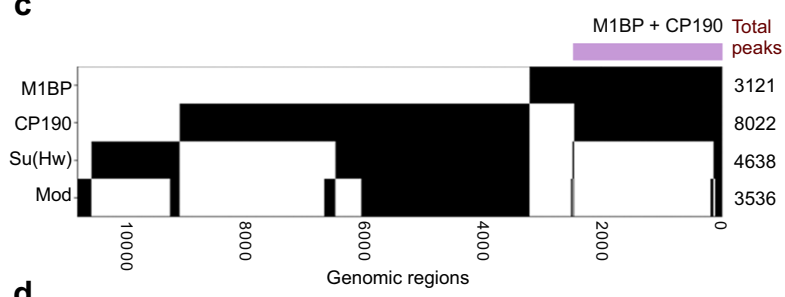

d

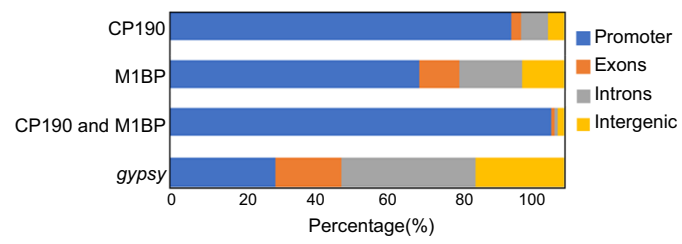

$\mathbf{f}$

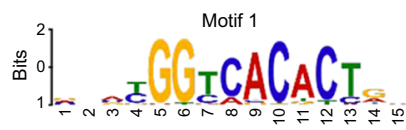

b

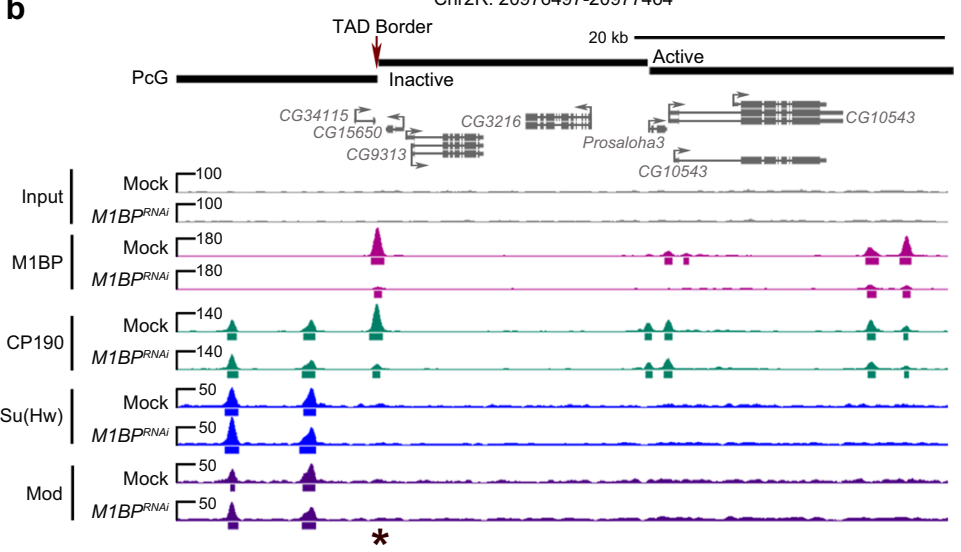

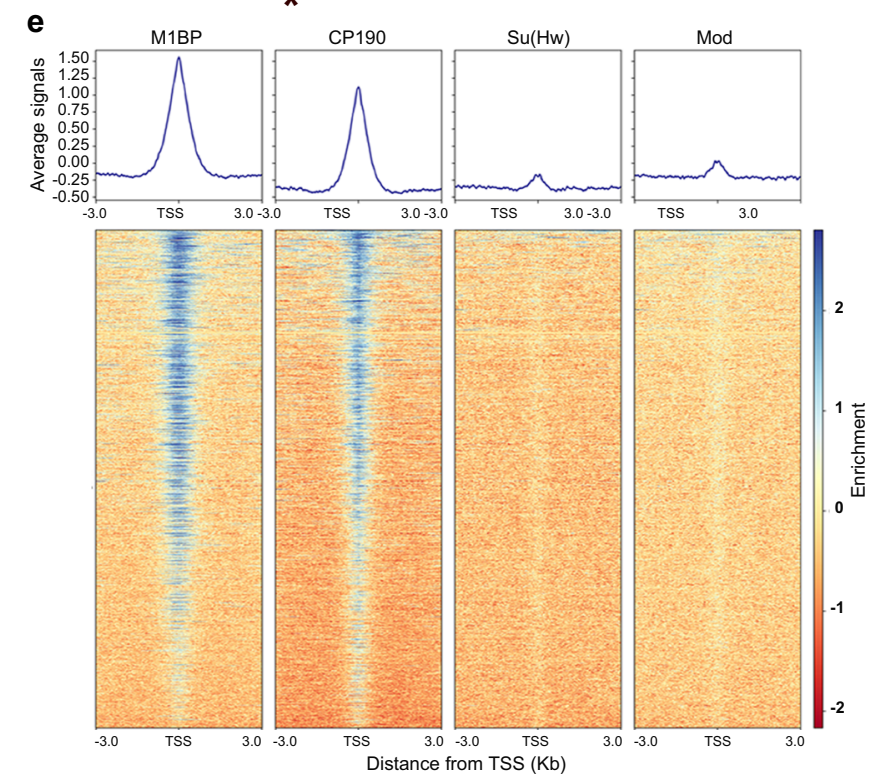

Fig. 3 M1BP extensively colocalizes specifically with CP190 throughout the genome. a Western blotting of total lysates from Kc control and M1BP knockdown cells showing knockdown efficiency of M1BP and no effects on protein levels of insulator proteins, with Pep as loading control. Samples from the same experiment were run on different lanes of the same gel for proteins with similar molecular weights. Western blotting was performed for three independent biological replicates with similar results. b Screenshot example of ChIP-seq profiles showing in Kc cells M1BP co-localizing with CP190 but not $\mathrm{Su}(\mathrm{Hw})$ or Mod(mdg4)67.2. Asterisk indicates a particular peak of interest measured in Fig. 6c, site 7. c Binary heatmap of M1BP, CP190, Mod(mdg4)67.2, and $\mathrm{Su}(\mathrm{Hw})$ binding sites in control cells ordered by supervised hierarchical clustering. Each row represents a single independent genomic location, and a black mark in a column represents the presence of a particular factor: 2461 (79\%, purple bar) peaks of 3121 total M1BP peaks overlap with CP190, 163 (5.2\%) M1BP peaks overlap with Su(Hw) peaks, and 217 (7\%) M1BP peaks overlap with Mod(mdg4)67.2. d Bar plot shows distribution of M1BP, CP190, and gypsy (Su(Hw)/Mod(mdg4)67.2/CP190 all present) binding sites with respect to genomic features. e Heatmaps of M1BP, CP190, Su(Hw), and Mod (mdg4)67.2 peaks in control cells sorted independently by decreasing average ChIP-seq signals normalized to inputs. Reads are centered on 6307 TSSs containing Motif 1 site. The horizontal axis corresponds to distance from TSS with Motif 1 site. $\mathbf{f}$ Motif 1 consensus sequence.

CP190 binding, but not $\mathrm{Su}(\mathrm{Hw})$ or $\mathrm{Mod}(\mathrm{mdg} 4) 67.2$ binding, are enriched at transcription start sites (TSS) (Fig. 3e). As expected, we verified that these promoters frequently harbor Motif 1 consensus sequence ${ }^{30,38}$ (Fig. $3 \mathrm{f}$ and Supplementary Data, 54\% of M1BP, 35\% of CP190, and 54\% co-occupied promoter peaks). Therefore, our ChIP-seq results indicate that M1BP colocalizes primarily with CP190 throughout the genome, particularly at Motif 1-containing promoters.

M1BP and CP190 regulate transcription of a similar gene set. Given that M1BP and CP190 colocalize extensively at promoters enriched for the presence of Motif 1, we compared how each factor affects transcription genome-wide. We performed neuRNA-seq after a $1 \mathrm{~h}$ pulse labeling of Kc cells to examine newly synthesized transcripts in mock transfected control cells versus knockdown of $M 1 B P, C p 190$, or $\bmod (\operatorname{mdg} 4)$ treatment specific for the $67.2 \mathrm{KDa}$ isoform. We found that depletion of M1BP results in upregulation of nascent transcription of 1315 genes and downregulation of 607 genes (Fig. 4a). Importantly, we found that only $22 \%$ of promoters of upregulated genes (Fisher's exact tests [FET], $P=1.6 \mathrm{e}-02$, odds ratio $=0.8)$ but $46 \%$ of promoters of downregulated genes harbor M1BP chromatin binding based on ChIP-seq analysis in the control condition (FET, $P<2.2 \mathrm{e}-16$, odds ratio $=2.8$ ). These results are consistent with an earlier report that M1BP is mainly directly involved in transcriptional activation ${ }^{30}$. Likewise, CP190 binds promoters of only $40 \%$ of genes that are upregulated after M1BP knockdown (FET, $P=7.3 \mathrm{e}-02$, odds ratio $=0.9$ ), but CP190 binding is highly enriched at gene promoters that are downregulated $(76 \%$, FET, $P<2.2 \mathrm{e}-16$, odds ratio $=4.4)$ resulting from M1BP depletion. Taken together, these results suggest that CP190 may cooperate 
a

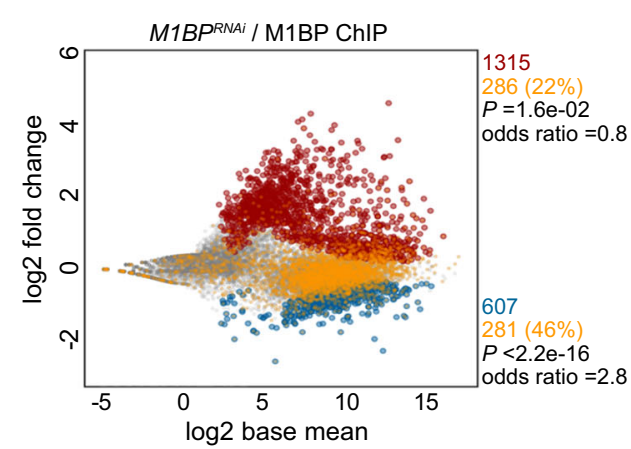

C

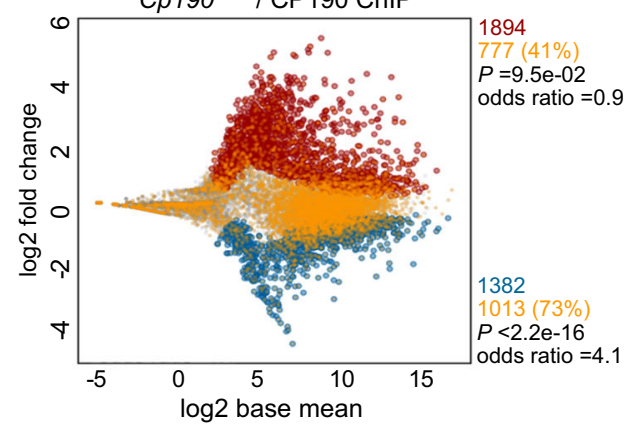

e

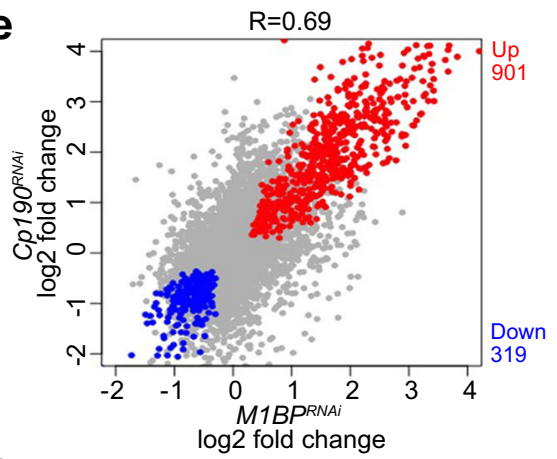

g

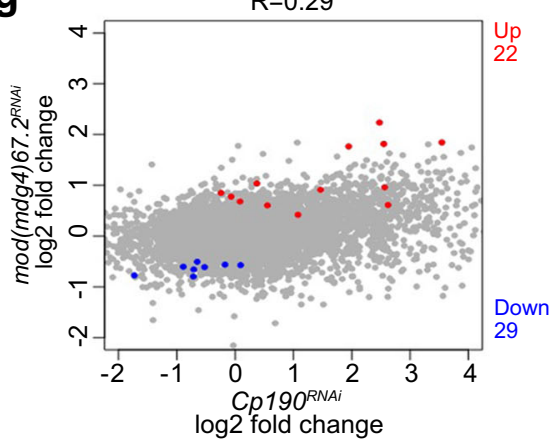

b

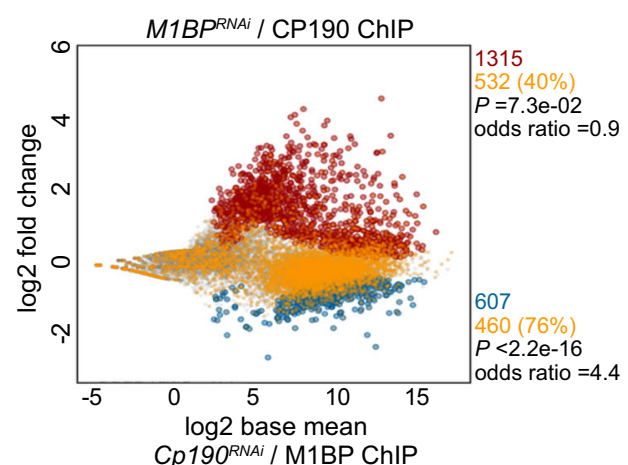

d

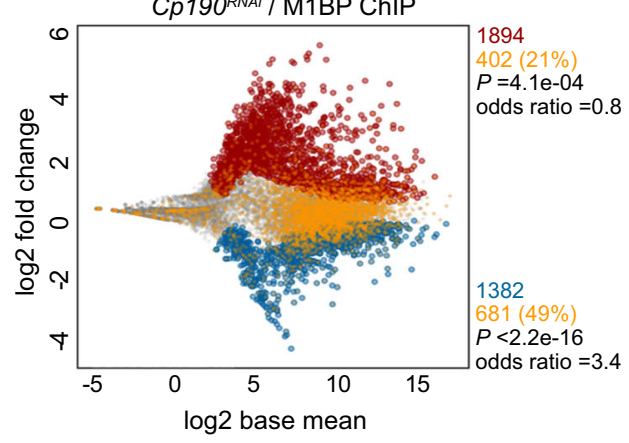

f

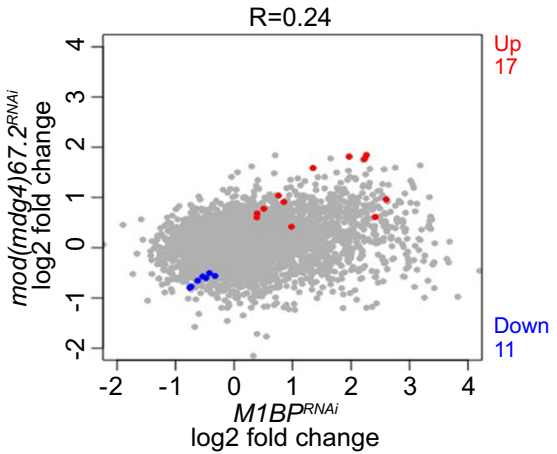

h

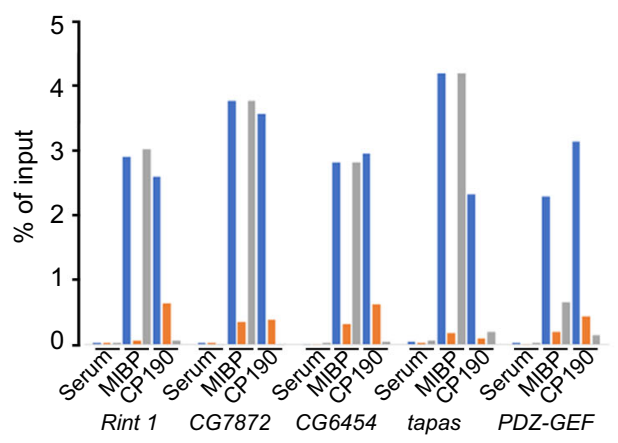

Mock

$M 1 B P^{R N A i}$

Cp190 RNAi

Fig. 4 M1BP and CP190 transcriptionally regulate a common set of genes. a, b MA plots showing changes in neuRNA levels upon depletion of M1BP. Statistically significant changes include 1315 upregulated genes (red) and 607 downregulated genes (blue) using $P_{\text {adj }}<0.05$. Unchanged genes are indicated in gray. Gene promoters containing M1BP peaks (a) or CP190 peaks (b) are additionally colored yellow. Two-sided Fisher's exact test was used. c, d MA plots showing neuRNA-seq affected genes after depletion of CP190. Significantly upregulated genes (1894, red) and downregulated genes (1382, blue) are shown. Genes containing CP190 (c) or M1BP (d) at their promoters are shown in yellow. Fisher's exact test (two-sided) was used. e-g Scatter plots comparing neuRNA-seq profiles of M1BP and Cp190 knockdowns (e), M1BP and mod(mdg4) knockdowns (f), or Cp190 and mod(mdg4) knockdowns (g). Pearson's $R$ corresponds to correlation coefficient between two profiles. Common upregulated genes are indicated in red, and common downregulated genes are indicated in blue. $\mathbf{h}$ Differentially decreased CP190 peaks in M1BPRNAi associated with the promoter of downregulated genes affected in both M1BP and Cp190 knockdowns are verified by ChIP-qPCR. Percent input DNA precipitated is shown for each primer set. Average values from $n=2$ biological replicates measured using four technical replicates are shown. Detailed description of each site is summarized in Supplementary Table 5, and $C_{t}$ values are listed in Source Data 7. 
a

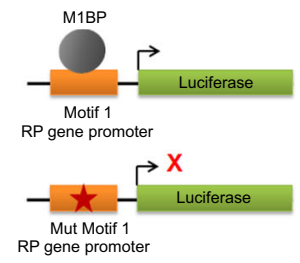

c

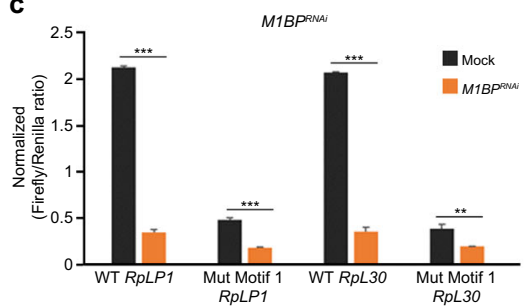

b

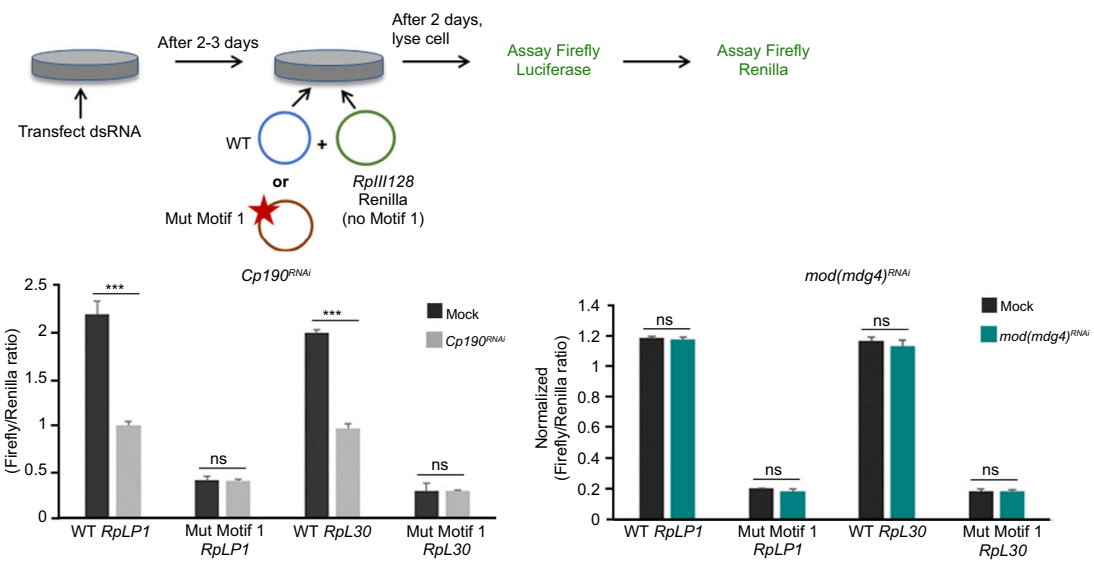

Fig. 5 M1BP and CP190 both activate Motif 1-containing gene promoters. a Schematic diagram of plasmids containing wild-type and Motif 1 mutants of ribosomal protein (RP) gene promoter driving luciferase reporters. b Schematic diagram showing RP gene luciferase reporter assay. Rp/II128 promoter lacks Motif 1 and serves as a transfection control. c Firefly/Renilla luciferase ratio of relative light unit measurements. Experiments are performed in indicated knockdown conditions. Data represented as mean \pm SD from $n=3$ biological replicates, and $P$ values were analyzed by two-tailed unpaired $t$-test. WT RpLP1 Mock vs M1BPRNAi; Mut Motif 1 RpLP1 Mock vs M1BPRNAi; WT RpLP30: Mock vs M1BPRNAi; WT RpLP1 Mock vs Cp190RNAi; and WT RpLP30 Mock vs Cp190RNAi all $P=0.0001$, Mut Motif 1 RpLP30 Mock vs M1BPRNAi $P=0.0017$, Mut Motif 1 RpLP1 Mock vs Cp190RNAi $P=0.73$, Mut Motif 1 RpLP30 Mock vs $C p 190^{R N A i} P=0.89$, WT RpLP1 Mock vs mod(mdg4) ${ }^{R N A i} P=0.49$, Mut Motif 1 RpLP1 Mock vs mod(mdg4) ${ }^{R N A i} P=0.16$, WT RpLP30 Mock vs $\bmod (\operatorname{mdg} 4)^{R N A i} P=0.28$, Mut Motif 1 RpLP30 Mock vs $\bmod (\operatorname{mdg} 4)^{R N A i} P=0.98$

with M1BP to activate transcription (Fig. $4 \mathrm{~b}$ and Supplementary Fig. 4a).

Intriguingly, we found that depletion of CP190 results in similar changes in nascent transcript levels compared to depletion of M1BP. After Cp190 knockdown, 1894 genes are upregulated, and 1382 genes are downregulated (Fig. 4c). Overall, the extent of nascent transcription changes is correlated with that of $M 1 B P$ knockdown $(R=0.69)$, with 901 upregulated and 319 downregulated genes in common (Fig. 4e). In contrast, knockdown of $\bmod (m d g 4)$ resulted in little overlap of nascent transcription changes compared to $M 1 B P$ knockdown $(R=0.24$, Fig. $4 \mathrm{f})$ or to Cp190 knockdown $(R=0.29$, Fig. 4g). Importantly, CP190 binding at the promoter in the control condition is also enriched at downregulated genes $(73 \%$, FET, $P<2.2 \mathrm{e}-16$, odds ratio $=4.1)$ but not upregulated genes $(41 \%$, FET, $P=9.5 \mathrm{e}-02$, odds ratio $=$ 0.9) after CP190 depletion (Fig. 4c). M1BP is also associated with promoters of downregulated genes (49\%, FET, $P<2.2 \mathrm{e}-16$, odds ratio $=3.4)$ but not upregulated genes $(21 \%$, FET, $P=4.1 \mathrm{e}-04$, odds ratio $=0.8$ ) after Cp190 knockdown (Fig. 4d). Finally, we verified that both M1BP plus CP190 binding together are also similarly statistically enriched predominantly at downregulated genes in either M1BP $(43 \%$, FET, $P<2.2 \mathrm{e}-16$, odds ratio $=2.9)$ or Cp190 knockdown condition (44\%, FET, $P<2.2 \mathrm{e}-16$, odds ratio =3.3) (Supplementary Fig. 4), suggesting a direct and specific relationship between CP190 and M1BP in transcriptional activation in particular. Although M1BP and CP190 appear to negatively regulate a similar set of genes, neither M1BP nor CP190 binding is enriched at the promoters of upregulated genes; therefore, these effects are likely to be indirect. Finally, we verified the association of M1BP and CP190 at promoters of several common downregulated genes dependent on M1BP and CP190 using directed ChIP-qPCR (Fig. 4h).

M1BP and CP190 facilitate Motif 1-dependent gene expression. To investigate whether CP190 is important for expression of genes dependent on Motif 1, we performed plasmid-based luciferase reporter assays in transfected Kc cells. We monitored luciferase expression driven by Motif 1-containing RpLP1 or $R p L 30$ promoters relative to Renilla, driven by the Motif 1 -independent
RpIII128 promoter, and expressed on a co-transfected control plasmid (Fig. 5a, b). Cells were mock transfected or knocked down for M1BP, Cp190, or $\bmod (m d g 4)$. Both M1BP and CP190 depletion decreased Motif 1-dependent $R p L P 1$ and $R p L 30$-driven luciferase expression, while mod(mdg4)67.2 knockdown had no effect (Fig. 5c and Supplementary Fig. 5). Importantly, Cp190 knockdown did not affect luciferase expression from constructs driven by $R p L P 1$ or RpL30 promoters that harbor point mutations in Motif 1 that abolish M1BP binding ${ }^{30,31}$. These results suggest that CP190 is important for Motif 1-dependent expression and raise the possibility that CP190 may also affect M1BP association with Motif 1containing promoters.

CP190 contributes to M1BP chromatin association. In order to test whether CP190 does indeed play a role in recruitment of M1BP to chromatin, we performed ChIP-seq of M1BP after depletion of CP190 in Kc cells. Western blot analysis illustrated that knockdown of Cp190 successfully reduced CP190 levels but did not have any effect on M1BP protein levels (Fig. 6a). In addition, we observed no major effect on cell proliferation or indication of mitotic arrest (Supplementary Fig. 2c-e). Using ChIP-seq, we verified substantial reduction of CP190 chromatin association (Fig. 6b). In order to identify statistically significant signal loss of called peaks by ChIP-seq, we applied the DiffBind algorithm ${ }^{39}$ at $P$ value $<0.05$ and identified $806 \mathrm{M} 1 \mathrm{BP}$ sites $(22 \%$ of total) with significantly decreased M1BP binding after CP190 depletion. This corresponds to loss of M1BP binding at $18 \%$ of co-occupied sites. The majority (81\%) of reduced M1BP peaks colocalize with CP190 binding in the control condition, suggesting that many effects are direct and that M1BP binding at these sites is facilitated by CP190. We validated six CP190dependent M1BP sites by directed ChIP-qPCR (Fig. 6c, sites 1-5) and another six sites that are bound by both M1BP and CP190 at which M1BP remains unaffected after CP190 depletion (Fig. 6c, sites 6-11, and Supplementary Fig. 4d). These data suggest that CP190 affects recruitment of M1BP to a subset of its binding sites within the genome.

We next examined the genome-wide relationship between both gene expression and M1BP chromatin association that is 
a

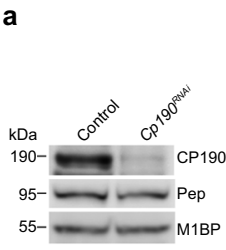

b

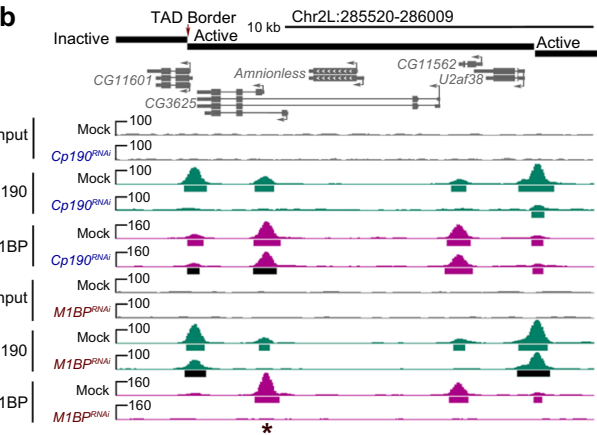

c

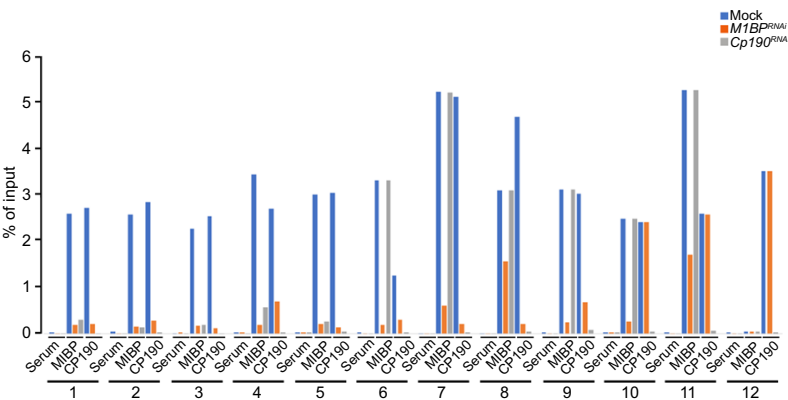

d

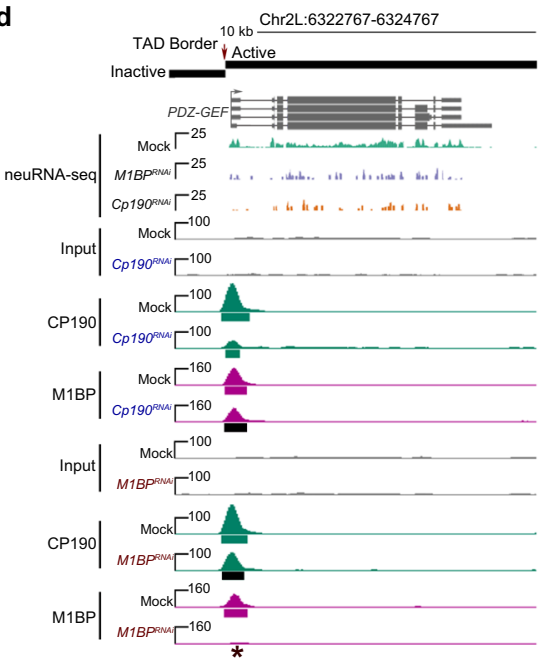

e
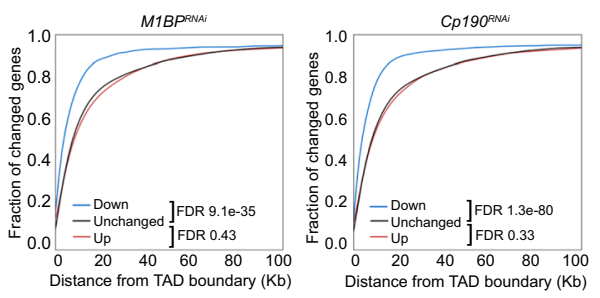

\begin{tabular}{|c|c|c|c|}
\hline & & Mediar & nce $(\mathrm{Kb})$ \\
\hline Knockdown & Gene changes & Affected & Unchanged \\
\hline \multirow{2}{*}{ M1BPRNAi } & Down & 2.9 & 6.6 \\
\hline & Up & 6.7 & 6.6 \\
\hline \multirow{2}{*}{ Cp190 PNAi } & Down & 3.0 & 6.8 \\
\hline & Up & 7.0 & 6.8 \\
\hline
\end{tabular}

f

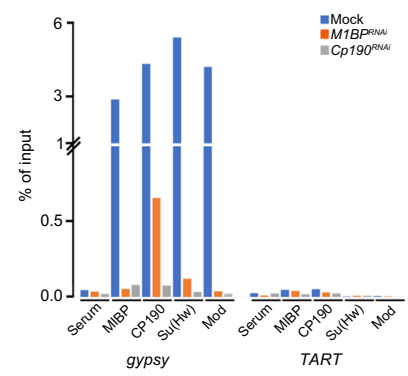

Fig. 6 M1BP association with chromatin is facilitated by CP190 at a subset of sites. a Western blotting of total lysates from Kc control and Cp190 knockdown cells with Pep used as loading control. This experiment was performed for three independent biological replicates with similar results. b Example screenshot of ChIP-seq profiles for lost CP190 peak in M1BPRNAi and lost M1BP peak in Cp190 ${ }^{R N A i}$ are shown. Asterisk indicates the particular interdependent peak measured in (c), site 1. Called ChIP-seq peaks in either knockdown condition shown in black are significantly decreased in the knockdown of the opposite factor. c Differentially bound M1BP and CP190 ChIP-seq peaks in M1BP knockdown and Cp190 knockdown validated by ChIPqPCR. Validation of selected differentially decreased peaks of M1BP and CP190 (sites 1-5) and negative control sites (sites 10 and 11). Validation of decreased peaks of CP190 (sites 6-9) and negative control site 12. Percentage input chromatin DNA precipitated is shown for each primer set, and average values from $n=2$ biological replicates measured using four technical replicates are plotted. Detailed description of each site labeled is summarized in Supplementary Table 5 and $C_{t}$ values are available in Source Data 7. d Example screenshot showing promoter association of M1BP and CP190 is interdependent for downregulated genes in either knockdown of M1BP or Cp190. In most cases, both proteins localize at TAD borders. For simplicity, only replicate 1 of mock, M1BPRNAi, and Cp190RNAi is shown for neuRNA-seq tracks. e Cumulative histograms of promoter distance from closest TAD border classified by change in nascent expression in M1BP (left) or Cp190 (right) knockdown cells. Downregulated (blue), upregulated (red), or unchanged (black) genes are indicated. Table indicates the median distance from closest TAD border for down- and upregulated genes compared to unchanged genes in either knockdown condition. Mann-Whitney $U$ test for each set of changed genes against unchanged genes are shown. Analysis of TADs classified by chromatin state is shown in Supplementary Fig. 6. f Percentage of precipitated input chromatin DNA from ChIPqPCR of M1BP, Su(Hw), Mod(mdg4)67.2, and CP190 at Su(Hw)-binding sites of gypsy or TART transposon sites as a negative control in Kc cells either mock-treated or subjected to M1BPRNAi or CP190RNAi. Average of $n=2$ biological replicates measured using four technical replicates are plotted. $C_{t}$ values are provided in Source Data 7.

dependent on CP190. We found that CP190-dependent M1BP binding sites are enriched at genes that require CP190 for full expression (Fig. 6 d, $9 \%$ of genes, FET, $P=1.7 \mathrm{e}-10$, odds ratio $=$ 2.0) but not for genes that are upregulated when CP190 is depleted ( $5 \%$ of genes, FET, $P=7.0 \mathrm{e}-01$, odds ratio $=0.9$ ). Taken together, CP190-dependent reduction of M1BP at these sites may culminate in a reduction of gene expression.

CP190 binding at some sites is dependent on M1BP. We next tested the opposite scenario: the non-exclusive possibility that M1BP affects CP190 recruitment throughout the genome. We thus performed ChIP-seq analysis of M1BP or CP190 in mock-treated versus M1BP-depleted Kc cells. Using the DiffBind algorithm, we identified 2018 CP190 peaks (25\% of total) that were reduced after M1BP depletion (Fig. 3b), and $34 \%$ of cooccupied sites show a decrease of CP190 binding. Again, we found that decreased CP190 peaks overlapped with M1BP binding in the control condition in a large proportion of cases $(42 \%$, FET, $P<$ $2.2 \mathrm{e}-16$, odds ratio $=2.0)$, suggesting that direct effects are observed. We validated a decrease of both CP190 and M1BP binding at co-occupied sites (sites 1-5) in M1BP knockdown using directed ChIP-qPCR (Fig. 6c and Supplementary Table 5). We also verified one CP190 binding site lacking M1BP that remained unchanged after M1BP depletion (Fig. 6c, site 12, Supplementary Fig. 4c, and Supplementary Table 5).

We then compared the genome-wide relationship between M1BP-dependent CP190 chromatin association and M1BP- 
dependent changes in nascent transcription. M1BP-dependent CP190 binding sites are enriched at promoters of genes that require M1BP for full expression (19\% of genes, FET, $P=$ $4.3 \mathrm{e}-09$, odds ratio $=1.9)$ but not genes that are upregulated in $M 1 B P$ knockdown ( $10 \%$ of genes, FET, $P=4.6 \mathrm{e}-01$, odds ratio $=$ 0.9). These results suggest that M1BP directly facilitates CP190 recruitment throughout the genome, and loss of these factors may manifest in reduced gene expression.

Effects of M1BP depletion occur near TAD borders. Because both M1BP and CP190 are enriched at promoters of genes located near TAD borders throughout the genome, we wanted to verify whether M1BP and CP190 activate transcription in proximity of TAD borders. We examined the distance from the promoter to the nearest TAD border for either upregulated or downregulated genes in either M1BP or CP190 knockdown cells and found that genes that require M1BP (false discovery rate [FDR] 9.1e-35) or CP190 (FDR 1.3e-80) for full activation are positioned significantly closer to Kc TAD borders compared to unchanged genes (Fig. 6e). In contrast, genes that are upregulated after M1BP (FDR 0.43) or Cp190 (FDR 0.33) knockdown are located a similar distance from TAD borders compared to unaffected genes. Similar trends are observed for genes located in all four classes of TADs (active, inactive, PcG, and HP1, Supplementary Fig. 6) as previously defined ${ }^{33}$. Overall our observations suggest that $\mathrm{M} 1 \mathrm{BP}$ and CP190 promote transcription near TAD borders genome-wide independently of TAD chromatin state.

M1BP and CP190 promote gypsy complex recruitment. Since M1BP rarely overlaps with $\mathrm{Su}(\mathrm{Hw})$ and $\operatorname{Mod}(\mathrm{mdg} 4) 67.2$ across the genome, we wanted to determine the mechanism by which M1BP promotes gypsy insulator activity in the context of the retrotransposon. To this end, we examined whether M1BP is also responsible for CP190 recruitment to gypsy retrotransposon sites. Interestingly, we did detect substantial M1BP chromatin association along with the three core insulator components at the 12 $\mathrm{Su}(\mathrm{Hw})$-binding sites of the gypsy retrotransposon in Kc cells (Fig. 6f). No binding of M1BP or core insulator proteins is observed at TART, another element found at multiple locations throughout the genome. After depletion of either M1BP or CP190, chromatin association of all four factors is dramatically reduced at the gypsy insulator binding site. These results are consistent with a direct effect of M1BP on gypsy chromatin insulator activity and on the recruitment of the core insulator components.

Depletion of M1BP alters formation of insulator bodies. Since M1BP promotes core complex recruitment to gypsy insulator sites as well as enhancer blocking and barrier activities, we examined the effect of M1BP on the nuclear localization of gypsy insulator bodies, of which proper formation correlates with insulator activities. We performed whole-mount immunostaining of dissected brains and imaginal discs of third instar larvae using antibodies against CP190 to detect insulator body localization. In the control line, approximately one insulator body per focal plane was observed in brain optic lobe, eye, leg, and wing discs (Fig. 7a). In contrast, multiple smaller insulator bodies were observed in $M 1 B P^{R N A i}$ driven by Act5C-Gal4, and the differences in number and size of insulator bodies are statistically significant (Fig. 7b, c). Moreover, the combined size of total insulator bodies per nucleus increased significantly in M1BP-depleted compared to control cells (Fig. 7d). These results indicate that M1BP ubiquitously affects localization of insulator bodies.
M1BP regulates local genome compaction. Changes observed in insulator body organization prompted us to examine whether M1BP or CP190 may affect global nuclear organization. Therefore, we examined the 3D localization of chromosome territories (CTs) for chromosome arms $2 \mathrm{~L}$ and 2R using Oligopaint FISH in Kc control versus M1BP or Cp190 knockdown cells. No significant differences in territory volume relative to nuclear volume, $\mathrm{CT}$ contact, or CT intermingling were observed (Supplementary Fig. 7). Furthermore, we analyzed the relative spatial positioning of two $50 \mathrm{~kb}$ loci (probes $\mathrm{A}$ and $\mathrm{B}$ ) located $\sim 3.1 \mathrm{Mb}$ apart on chromosome $3 \mathrm{~L}$ and found no difference in the distance between these regions after M1BP- or CP190 depletion (Supplementary Fig. 8). We conclude that M1BP and CP190 do not affect largescale genome organization.

To investigate if local compaction is altered after M1BP or CP190 depletion, we next measured the distance between two more closely spaced regions ( $\sim 4 \mathrm{~kb}$ apart) in distinct TADs separated by M1BP and CP190 binding sites. These regions are also of interest because M1BP- and CP190-dependent nascent transcription is observed within this vicinity (Supplementary Fig. 9a). Examination of these two $30 \mathrm{~kb}$ probes $(\mathrm{C}$ and $\mathrm{D})$ in control and M1BP- or CP190-depleted cells showed that depletion of M1BP but not CP190 resulted in a statistically significant decrease in the distance between probes (Supplementary Fig. 9c, e). Moreover, we examined a second pair of probes (E and F) located $\sim 13 \mathrm{~kb}$ apart spanning the previously characterized Nhomie and Homie insulator sites respectively ${ }^{40}$, which flank a Polycomb Group (PcG)-repressed TAD and also feature M1BP and CP190 binding as well as M1BP- and CP190-dependent nascent transcription (Supplementary Fig. 9b). The distance between these probes was also significantly decreased in M1BPbut not CP190-depleted cells (Supplementary Fig. 9d, e).

Changes in local genome compaction in M1BP-depleted cells could be a result of compaction specifically at TAD borders. To test this possibility, we measured the distances between three 32 $\mathrm{kb}$ probes each spaced $15 \mathrm{~kb}$ apart with two probes ( $\mathrm{G}$ and $\mathrm{H}$ ) inside a large inactive TAD and the third (probe I) spanning the entirety of a flanking PcG TAD (Fig. 8a). We found that distances between G-H and H-I significantly decreased to a similar extent in M1BP- but not CP190-depleted cells, indicating that increased compaction is not exclusively observed across this TAD border but also occurs within this large inactive TAD (Fig. 8b, c). Two additional sets of three $32 \mathrm{~kb}$ probes (J, K, L and $\mathrm{M}, \mathrm{N}, \mathrm{O}$ ) similarly designed to adjacent TADs likewise show increased inter- and intra-TAD interactions in M1BP- but not CP190depleted cells (Fig. 8d-i), suggesting that increased local compaction is not limited to TAD borders, specific classes of TADs, or particular configurations.

Loss of M1BP reduces chromatin accessibility near TAD borders. Increases in local compaction observed by Oligopaint FISH in M1BP-depleted cells motivated us to examine whether M1BP affects chromatin accessibility genome-wide. We therefore performed Assay for Transposase-Accessible Chromatin (ATACseq) in mock-treated or M1BP-depleted Kc cells. We identified 21,355 high-confidence ATAC-seq peaks in the control sample but only 17,585 ATAC-seq peaks in M1BP-depleted cells, indicating substantial overall loss of genome-wide chromatin accessibility and increased chromatin compaction after loss of M1BP. Almost all M1BP ChIP-seq peaks (92\%) overlap accessible sites (Fig. 9a), and the average ATAC-seq signal centered on these regions is reduced after M1BP-depletion (Fig. 9b), suggesting that M1BP is required to maintain open chromatin at these sites.

Consistent with M1BP mainly promoting chromatin accessibility, approximately half of M1BP binding sites show a reduction 

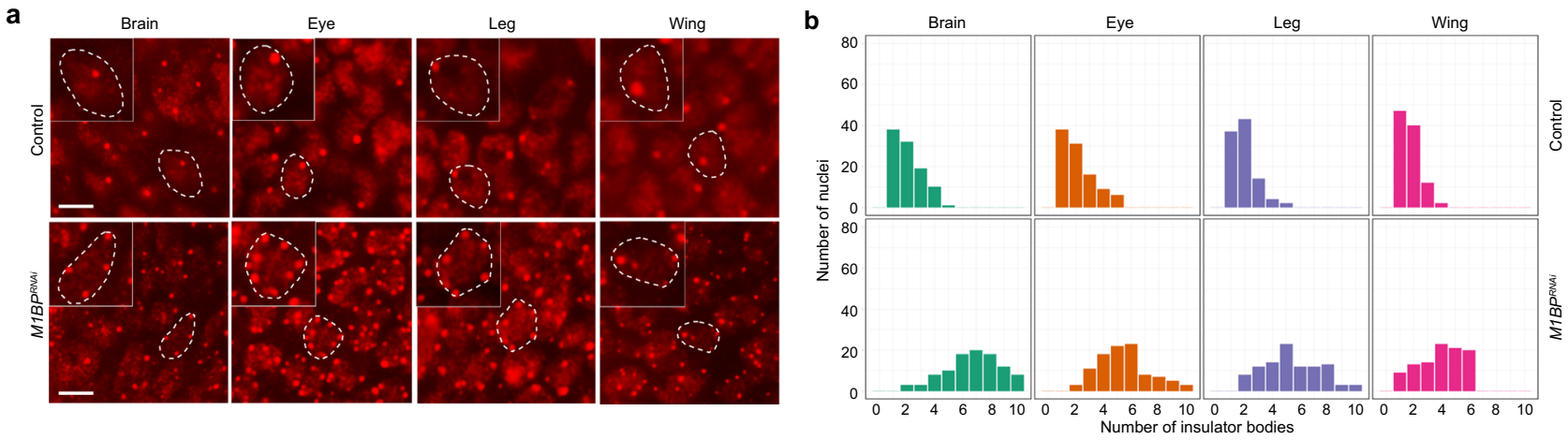

c
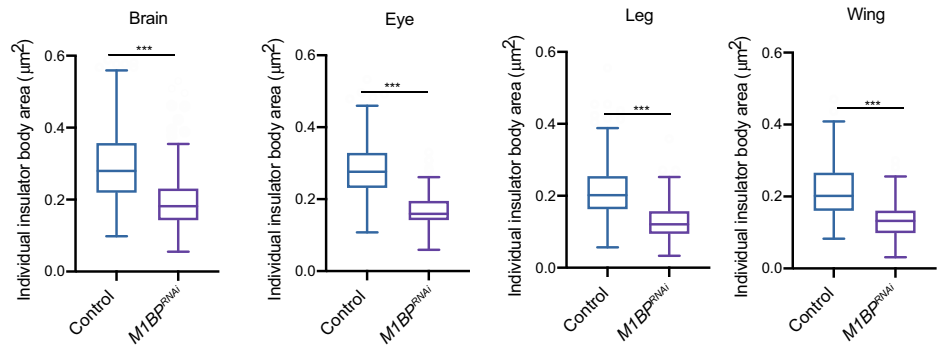

d
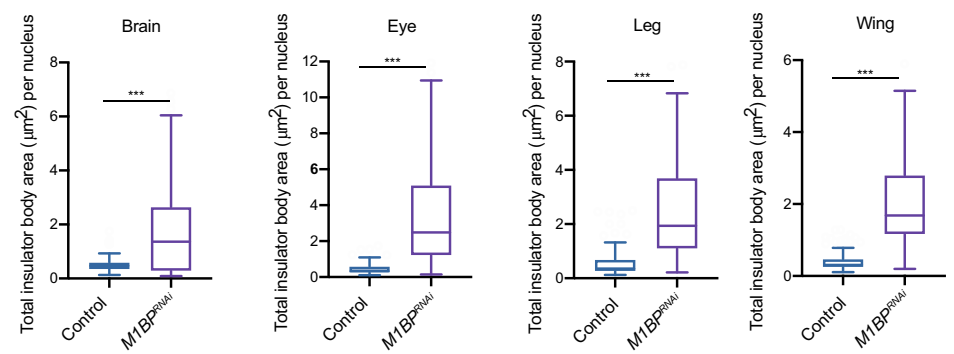

Fig. 7 Knockdown of M1BP alters nuclear organization of insulator bodies. a Epifluorescence imaging of insulator body localization using anti-CP190 in whole-mount brain, eye, leg, or wing imaginal disc tissues. M1BP knockdown is driven by Act5C-Gal4 driver. Insets show zoom of single nucleus outlined with dashed line in larger panel. Scale bars: $5 \mu \mathrm{m}$. Immunostaining experiments were performed three times with similar results. b Histograms showing the number of insulator bodies per nucleus in the experiment exemplified in (a). In all tissues, the number of insulator bodies is statistically significantly increased in M1BP knockdown (Kruskal-Wallis test; all Benjamini-Hochberg corrected $P<5 \times 10^{-17}, n=100$ ). c Area measurements of individual insulator bodies in brain, eye, leg, and wing imaginal disc tissues of control and M1BP knockdown larvae are shown. Bodies were measured (Tukey plots with outliers omitted, Mann-Whitney test $P<0.001, n=200$ ). d Area measurements of total insulator bodies per nucleus are shown (Tukey plots with outliers omitted, Mann-Whitney test $P<0.001)$; Brain: (Control $\left.n=108, M 1 B P^{R N A i} n=109\right)$, Eye: (Control $n=121$, M1BPRNAi $\left.n=168\right)$, Leg: (Control $n=107$, M1BPRNAi $n=$ 103), Wing: (Control $n=109$, M1BPRAi $_{n}=109$ ). Note that not all cells have discernible nuclear demarcations. $\mathbf{c}$, $\mathbf{d}$ Data are presented as boxplots where box represents the 25-75th percentiles and middle line is the median. The upper whisker extends from the hinge to the largest value no further than $1.5 \times$ IQR from the hinge (where IQR is the interquartile range), and the lower whisker extends from the hinge to the smallest value at most $1.5 \times$ IQR of the hinge, while data beyond the end of the whiskers are outlying points that are omitted from the plots.

of chromatin accessibility as defined by the DiffBind algorithm $(\mathrm{FDR}<0.05)$, whereas approximately $30 \%$ correspond to an increase (Fig. 9a). We also observed lower ATAC-seq signal at regions immediately upstream and at well-positioned nucleosomes downstream of the TSS of genes downregulated but not upregulated after depletion of M1BP (Fig. 9c-e), consistent with the previous finding that M1BP activates transcriptionally paused genes $^{30}$. Furthermore, sites with reduced chromatin accessibility overlap significantly specifically with decreased nascent expression (Supplementary Fig. 11a, b). Finally, reduced ATAC-seq signal is observed at TAD borders after M1BP-depletion (Fig. 9f), and reduced ATAC-seq peaks are positioned substantially closer to TAD borders than unchanged or increased peaks (Fig. 9g). In contrast, depletion of CP190 resulted in only mild changes in chromatin accessibility genome-wide (21,093 total ATAC-seq peaks) (Supplementary Fig. 11c-j). Taken together, these results show that loss of M1BP mainly results in reduced chromatin accessibility at its binding sites and in the vicinity of TAD borders across the genome, and these changes correlate with reduced transcriptional activation.

\section{Discussion}

Here we show that M1BP is required for proper gypsy insulator function and insulator body formation and that M1BP and CP190 together activate transcription at TAD borders. We found that M1BP physically associates with CP190 as well as core gypsy components and promotes enhancer blocking and barrier activities. Genome-wide M1BP colocalizes mainly with CP190 at Motif 1-containing promoters, which are enriched at TAD borders. M1BP is required for CP190 binding at many sites throughout the genome and vice versa, and loss of either factor reduces gene expression at TAD borders. M1BP is required for proper nuclear localization of insulator bodies, and loss of M1BP increases local genome compaction across TAD borders as well as within large TADs. Finally, M1BP promotes local chromatin accessibility at its 


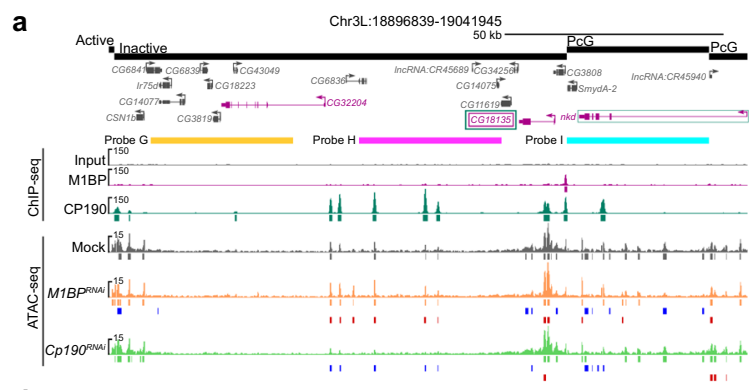

d
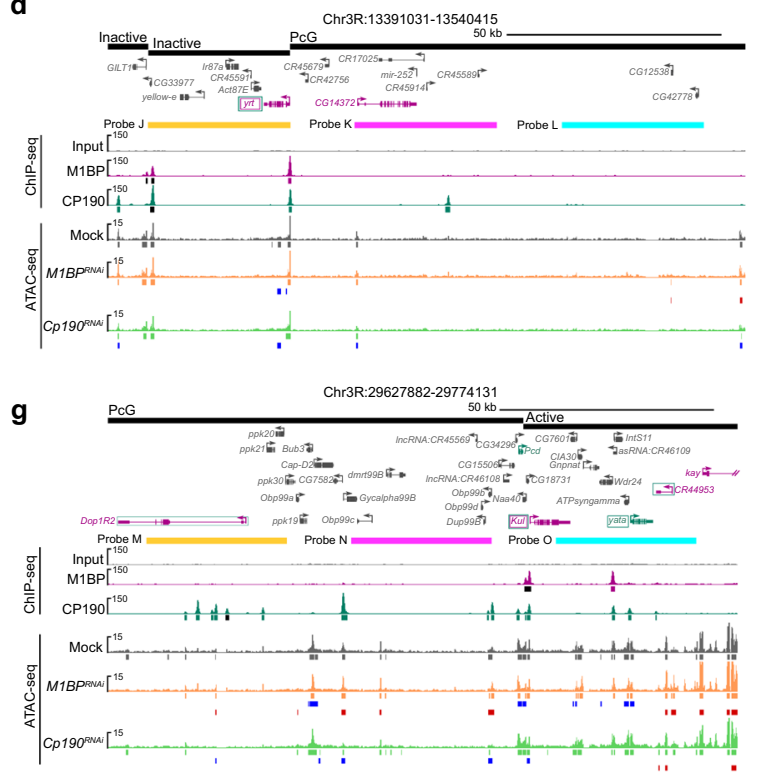

b

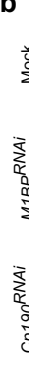

e

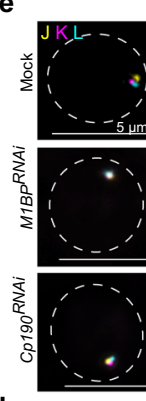

h

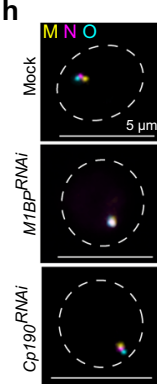

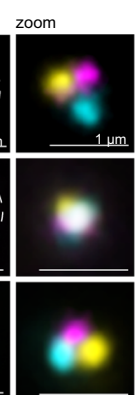
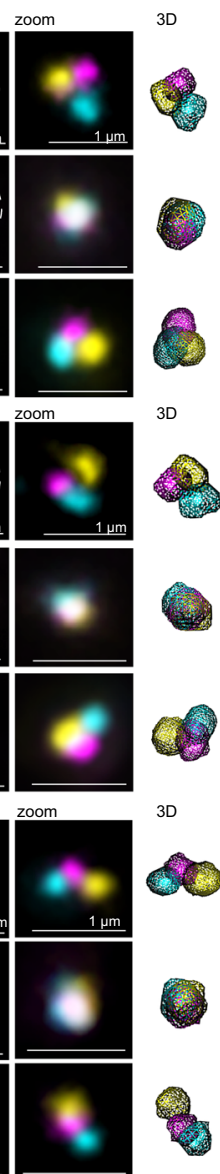

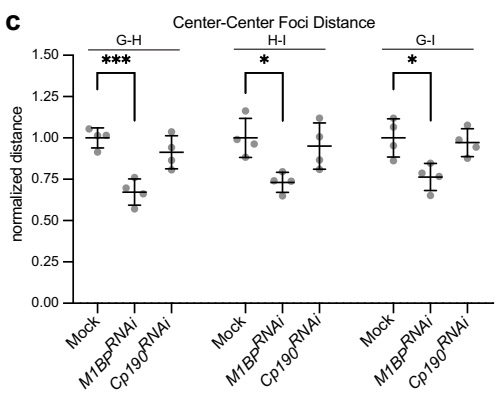

f

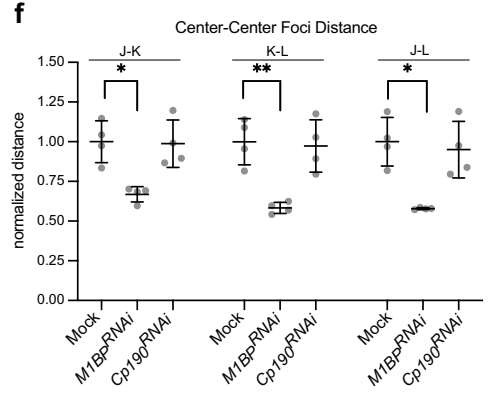

i

Fig. 8 Knockdown of M1BP increases local inter-TAD and intra-TAD genome compaction. a, $\mathbf{d}$, $\mathbf{g}$ Regions detected by $32 \mathrm{~kb}$ probes spaced $15 \mathrm{~kb}$ apart (a probes $\mathrm{G}, \mathrm{H}, \mathrm{l}$; d probes J, K, L; $\mathbf{g}$ probes $\mathrm{M}, \mathrm{N}, \mathrm{O}$ ). TADs with state classification, longest gene isoform, ChIP-seq signals, and called peaks (black significantly decreased in knockdown of opposite factor) are shown. ATAC-seq signals and called peaks, decreased peaks relative to mock (blue), and increased peaks (red) are shown. Upregulated genes in either M1BP or Cp190 knockdown are shown in purple or green, respectively. Downregulated genes in either M1BP or Cp190 knockdowns text outlined with a box are shown in purple or green, respectively. Note that nkd, Dop1R2, and CR44953 are upregulated in both knockdowns, and CG18135, yrt and Kul are downregulated in both knockdowns. b, e, h Left: representative nuclei labeled with probes in $\mathbf{a}, \mathbf{d}$, and $\mathbf{g}$, respectively. Max projection of approximately $5 \mathrm{Z}$ slices. Dashed line represents nuclear edge. Center: zoom of FISH signals. Right: TANGO 3D mesh rendering. $\mathbf{c}, \mathbf{f}, \mathbf{i}$ Dot plots showing average pairwise center-to-center distances between probes in $\mathbf{a}, \mathbf{d}$, and $\mathbf{g}$, respectively. Data represented as mean of all replicates (mid-line) \pm SD (error bars). Each dot represents the average of one replicate. Single-cell distances were normalized to nuclear radius before population averages were calculated. All averages were normalized to average of mock controls. Only cells in G1 were measured for these analyses. Data are from four biological replicates. See Supplementary Tables 10-12 for " $n$ " of cells examined per replicate. Unpaired $t$-test (two-tailed) of means before normalization to controls $\left({ }^{\star} P<0.05,{ }^{\star \star} P<0.01,{ }^{\star \star \star} P<0.001\right)$. Exact $P$ values can be found in Supplementary Table 13.

binding sites, including transcriptionally activated genes and regions near TAD borders. Taken together, our findings suggest that M1BP may play a role in $3 \mathrm{D}$ genome organization through a CP190- and transcription-dependent mechanism.

As M1BP is ubiquitously expressed throughout development, we observe effects on insulator activity and complex localization after M1BP depletion in all tissues and stages of development tested. M1BP associates physically with chromatin at the $\mathrm{Su}(\mathrm{Hw})$ binding sites of the gypsy insulator in conjunction with core insulator proteins, and M1BP is required for the binding of all three factors. These findings suggest that M1BP directly affects insulator activity by aiding the recruitment of gypsy core components to gypsy insulator sites, all three of which are required for proper insulator activity ${ }^{7,9,10}$. Interestingly, Motif 1 is not present at this sequence, and M1BP binding at this site is also dependent on the presence of CP190. One scenario is that binding of the two factors could be cooperative, and M1BP recruitment may additionally help stabilize the multimerization and/or higher order organization of insulator complexes. Consistent with this hypothesis, depletion of M1BP results in increased numbers of smaller insulator bodies, similar to the effect of complete loss of the BTB-containing core insulator protein $\operatorname{Mod}(\operatorname{mdg} 4) 67.2^{23,26}$. Another possibility is that depletion of M1BP results in cellular stress that induces insulator body formation. Since CP190 is a universal insulator protein in Drosophila, mislocalization of CP190 may result in, or at least serve as an indicator of, disrupted genome organization when M1BP is depleted.

Although M1BP physically interacts with each of the core gypsy insulator components, we observed that M1BP colocalizes mainly with just CP190 throughout the genome, particularly at Motif 1-containing promoters. Distinct binding of the transcriptional activator M1BP compared to $\mathrm{Su}(\mathrm{Hw})$ and $\mathrm{Mod}(\mathrm{mdg} 4) 67.2$ genomewide is not entirely surprising considering sub-stoichiometric levels of co-immunoprecipitation that could also reflect interaction off of chromatin. Furthermore, it has been observed that $\mathrm{Su}(\mathrm{Hw})$ binding can correlate with transcriptional repression rather than insulator activity, which may depend on the presence, or absence, of particular interacting proteins ${ }^{41}$. Importantly, recruitment of CP190 is 
a

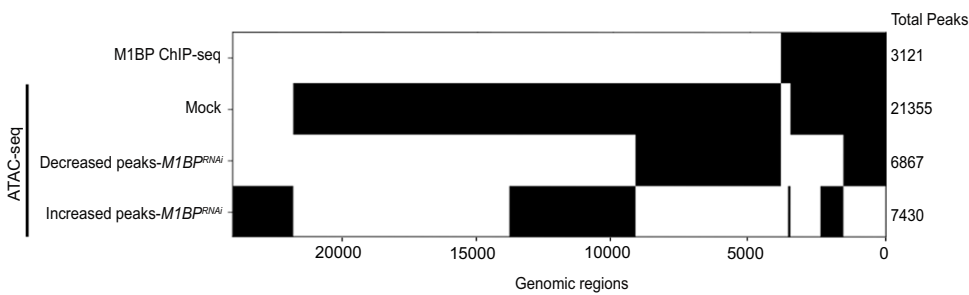

b

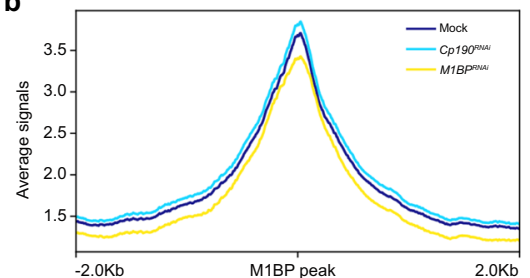

c

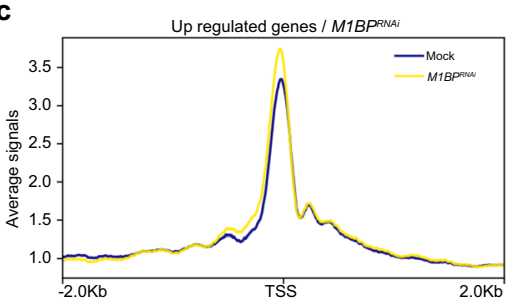

d

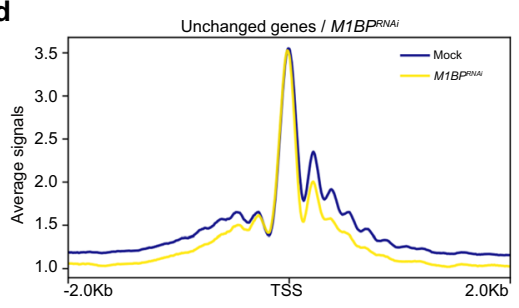

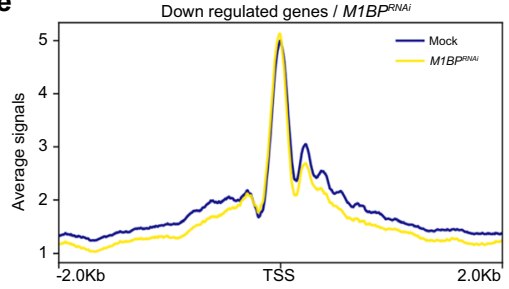

f

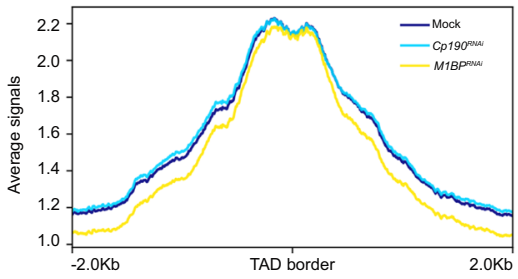

g

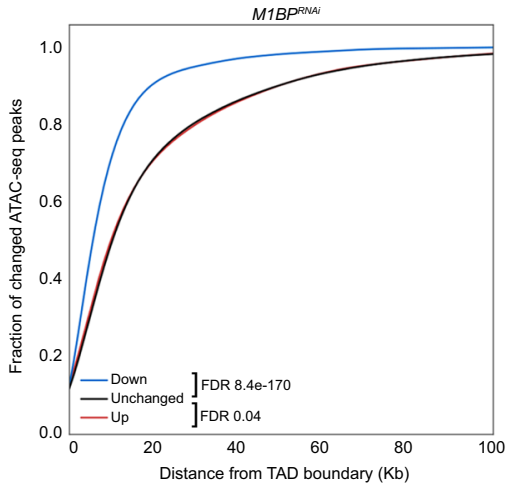

Fig. 9 Depletion of M1BP reduces the chromatin accessibility near TAD borders genome-wide. a Binary heatmap of M1BP ChIP-seq peaks, Mock ATACseq peaks, decreased and increased ATAC-seq peaks of M1BPRNAi compared to Mock ordered by supervised hierarchical clustering. Each row represents a single independent genomic location, and a black mark in a column represents the presence of a particular factor. b Average ATAC-seq tagmentation signals of Mock, M1BPRNAi and Cp190RNAi cells for a $4 \mathrm{~Kb}$. genomic window centered on M1BP-binding sites (ChIP-seq peaks). c Average ATAC-seq signals of Mock and M1BPRNAi cells for a $4 \mathrm{~Kb}$ genomic window centered on TSS of upregulated genes, $\mathbf{d}$ unchanged genes, and e downregulated genes in M1BP knockdown. $\mathbf{f}$ Average ATAC-seq signals of Mock, M1BPRNAi, and Cp190RNAi cells are plotted for a $4 \mathrm{~Kb}$ genomic window centered at TAD borders. g Cumulative histograms of ATAC-seq peak center distance from closest TAD border classified by change in ATAC-seq peaks in M1BP knockdown cells relative to Mock. Decreased (blue), increased (red), or unchanged (black) ATAC-seq peaks are indicated. Mann-Whitney $U$ test for each set of changed peaks against unchanged peaks is shown.

dependent on M1BP and vice versa at many co-occupied sites genome-wide. Why M1BP is only partially dependent on CP190 for binding is unclear, but these results are consistent with the known ability of M1BP to bind DNA directly ${ }^{30}$, whereas CP190 is believed to require interaction with a specific DNA-binding protein in order to associate with chromatin $8,17,18,42,43$. Aside from the insulator protein BEAF-32, which binds an AT-rich dual core sequence ${ }^{44}$, we did not generally observe a large extent of overlap between M1BP and other DNA-binding insulator proteins that have been shown to be involved in recruiting CP190 to DNA. Future studies may reveal a possible functional relationship between M1BP and BEAF-32 in insulator activity or regulation of gene expression.

Our results suggest that M1BP promotes gypsy insulator function through interaction with CP190 in a manner distinct from CP190 interaction with the zinc-finger DNA-binding protein CLAMP. We did not observe a large extent of genome-wide overlap between M1BP and CLAMP, a recently identified positive regulator of gypsy insulator activity ${ }^{16}$. The sequence binding specificity of CLAMP is similar to that of $\mathrm{GAF}^{45}$, while M1BP and GAF bind to and regulate distinct sets of promoters ${ }^{30}$. Although either CLAMP or M1BP depletion reduces gypsy enhancer blocking and barrier activities as well as alter insulator body localization, unlike M1BP, CLAMP depletion does not affect
CP190 chromatin association throughout the genome ${ }^{16}$. In fact, CP190 depletion had a substantial effect on CLAMP chromatin association, again suggesting that CP190 may affect the ability of certain DNA-binding proteins, including M1BP, to associate with chromatin through cooperative or higher order physical interactions.

Our results suggest that CP190 may play a more direct role in transcriptional regulation than previously appreciated, in part through interaction with M1BP. Genome-wide profiling studies have shown that CP190 preferentially associates with promoters genome-wide ${ }^{13,16,46}$. CP190 was found to be enriched particularly at active promoters and was shown to affect steady-state gene expression when depleted; however, direct and indirect effects as well as transcriptional and posttranscriptional effects could not be separated $^{46}$. In order to avoid the complication of interpreting steady-state gene expression profiles, we performed neuRNA-seq after either CP190 or M1BP depletion in order to measure newly synthesized transcripts. Intriguingly, nascent RNA expression profiles of CP190 or M1BP-depleted cells showed a remarkably high level of correlation. Since both M1BP and CP190 are particularly associated with promoters of genes that require either factor for adequate expression, it is likely that both factors mainly function in transcriptional activation rather than repression. 
M1BP was previously shown to activate transcriptionally paused genes $^{30}$, and our ATAC-seq analysis of M1BP-depleted cells supports this conclusion and further demonstrates that M1BP promotes chromatin accessibility surrounding the TSS. Interestingly, depletion of CP190 has no effect on promoter accessibility. Furthermore, we found that CP190 is specifically required for Motif 1-dependent expression of two previously characterized ribosomal protein genes. Whether TRF2 recruitment to Motif 1containing promoters is affected by CP190 as well as the precise mechanism by which CP190 contributes to M1BP-dependent transcription will be important topics of further study.

We found that genes that require M1BP and CP190 for adequate expression are frequently located at TAD borders, as are both proteins. It has previously been proposed that constitutively active transcription, particularly at/near TAD borders (also referred to as "compartmental domains"), may be a defining or at least key feature of overall genome organization in cells $4,6,35$ and throughout development ${ }^{5}$. CP190 was previously shown to be associated particularly with Motif 1 -containing promoters ${ }^{29}$, and CP190 was observed to be specifically enriched at TAD borders $^{34,35}$. Recently, Motif 1 was also found to be an enriched sequence at TAD borders present in $\mathrm{Kc}$ cells $^{33}$ and is apparent when TAD borders first appear in embryonic development ${ }^{5}$, findings consistent with these previous studies. A previous study of M1BP involvement in genome organization provided limited evidence using $\mathrm{Hi}-\mathrm{C}$ to suggest that chromosome intermingling may be increased after M1BP depletion ${ }^{33}$. However, the extent and duration of M1BP depletion in their study caused a major disruption of cell cycle and cellular growth, thus obscuring interpretation of those results. We did not observe changes in CT intermingling in our Oligopaint FISH experiments in M1BPdepleted G1 cells, nor did we observe any difference in distance between two distant regions on the same chromosome. Therefore, large-scale changes in genome organization were not observed after M1BP depletion.

We did observe increased local genome compaction after M1BP depletion. This finding led us to test whether TAD borders may be specifically disrupted, perhaps leading to fusion of neighboring TADs. Because our Oligopaint FISH probes are limited to a minimum of $30 \mathrm{~kb}$, we were restricted to intra-TAD analysis of larger TADs, which are typically lower in transcriptional activity (PcG, inactive, null). We found that increased compaction occurs both across TAD borders and within large TADs after M1BP depletion. These effects occur in the vicinity of altered local transcription and reduced chromatin accessibility particularly near TAD borders, suggesting that M1BP-dependent transcriptional changes might alter local chromatin structure that culminates in changes in genome compaction in surrounding regions not restricted to TAD borders. However, the extent of reduced chromatin accessibility observed in M1BP-depleted cells by ATAC-seq is modest relative to the genomic sequence space interrogated by FISH, suggesting that loss of accessibility likely does not directly explain increased local genome compaction. In contrast, CP190 depletion affected transcription to a similar degree, although not identically, yet did not result in changes in local compaction or extensive loss of chromatin accessibility genome-wide. These differences perhaps reflect the multifunctional nature of CP190 as a universal insulator protein contributing to opposing forces, or alternatively, effects on transcription in M1BP-depleted cells may be functionally unrelated to increased genome compaction. Our work shows the requirement of M1BP for accurate CP190 binding throughout the genome as well as gypsy-dependent chromatin insulator activity and nuclear localization through interaction with CP190 and other core insulator proteins. Overall, our results provide evidence that M1BP and CP190 at TAD borders and perhaps their ability to activate transcription of constitutively expressed genes located at TAD borders, combined with the capacity of M1BP to promote chromatin accessibility, may play a role in genome organization.

M1BP has been shown to activate transcription of genes at which RNA Pol II is transiently paused in the promoter proximal region, and these promoter regions may themselves possess insulator activity. Intriguingly, a previous study showed that stalled Hox promoters, including Motif 1-containing $A b d-B$, possess intrinsic enhancer blocking insulator activity ${ }^{47}$. However, the second paused promoter identified in this study, $U b x$, does not harbor Motif 1; thus, M1BP may not necessarily be involved in the enhancer blocking activity of all stalled promoters. Recently, it was shown that M1BP promoter binding can prime the recruitment of the Hox protein Abd-A to the promoter in order to release paused Pol II and activate transcription ${ }^{48}$. We find that M1BP is similarly required for CP190 recruitment at a large number of sites throughout the genome and thus propose that M1BP and CP190 are together required to maintain active gene expression near TAD borders. Active transcription and increased accessibility at these sites may be needed for higher order chromatin organization such as TAD insulation, formation of active compartmental domains, and/or proper local genome structure. Future studies will elucidate the precise mechanisms by which M1BP, CP190, and transcription contribute to higher order chromatin organization.

\section{Methods}

Drosophila strains. Fly lines were maintained on standard cornmeal medium at $25^{\circ} \mathrm{C}$. We used lines expressing dsRNA against $s u(H w)(10724 \mathrm{GD})^{24}$ and $M 1 B P$ $(110498 \mathrm{KK})$ from the Vienna Drosophila RNAi Center. $y, w[1118]$; $P\{a t t P, y[+], w$ [3'] (60100 KK) was used as a control for M1BP KK RNAi lines. Act5C-Gal4, Mef2 Gal4 and l(3)31-1-Gal4 driver lines were obtained from Bloomington Drosophila Stock Center. We scored the $c t^{6}$ phenotype on the first day after eclosion ${ }^{16,24}$. Number of male flies and percentage of male flies for each score are available in Source Data 7. UAS-luciferase constructs were inserted into the attP3 landing site using phiC31 site-specific integration ${ }^{49}$. Protein extracts from anterior thirds of larvae were used for western blotting. Embryos aged 0-24 h were collected from wild-type Oregon R raised at RT in population cages fed with yeast and molasses Embryos were dechorionated with $50 \%$ bleach, washed and stored at $-80^{\circ} \mathrm{C}^{50}$ to produce nuclear extracts.

Luciferase insulator barrier activity assay. Insulator barrier activity by luciferase assay was carried out using Bright-Glo ${ }^{\text {ms }}$ Luciferase Assay System (Promega) ${ }^{16,24}$. Individual male larvae were homogenized in $50 \mu \mathrm{L}$ of Glo Lysis buffer (Promega) and incubated at RT for $10 \mathrm{~min}$. Extracts were cleared from debris by centrifuga tion. Then, $20 \mu \mathrm{L}$ of soluble extract was dispensed in a 96-well white flat bottom plate (Costar), and the same volume of Bright-Glo luciferase reagent (Promega) was added to each well. Luciferase signal was quantified using a Spectramax II Gemini EM plate reader (Molecular Devices). Luciferase levels were measured for 12 individual whole third instar male for all genotypes indicated in a single panel simultaneously. Luciferase value was normalized to total protein of each larva determined by BCA reagent (Thermo Scientific). The relative luciferase activity of a population of a single genotype was aggregated into a box and whisker plot. Populations were compared with one-way ANOVA followed by a Tukey HSD post hoc test to obtain $P$ values for each pairwise comparison. The $P$ values for pairwise comparisons between the control and RNAi lines within both non-insulated and insulated groups are listed in Supplementary Table 4. All values of relative luciferase activity are available in Source Data 7.

Immunostaining of imaginal discs. Imaginal discs and brains were dissected from at least six larvae of each genotype following whole-mount staining methods ${ }^{28}$. Dissected tissues were fixed with $4 \%$ paraformaldehyde prepared in PBS-T (1X PBS, $0.1 \%$ Tween-20) for $20 \mathrm{~min}$ with rotation at RT. Tissues were washed $3 \times 5$ min with PBS-T and blocked with blocking solution (1X PBS, $0.3 \%$ Triton, $10 \%$ Normal Goat serum) for $2 \mathrm{~h}$. Tissues were immunostained with rabbit serum against CP190 in blocking solution overnight at $4{ }^{\circ} \mathrm{C}$. Tissues were washed $3 \times 20$ min with PBS-Tr (1X PBS, $0.3 \%$ Triton) at RT. Tissues were incubated with secondary antibody in blocking solution at $37^{\circ} \mathrm{C}$ for $2 \mathrm{~h}$. Then tissues were washed $2 \times 1$ h with PBS-Tr, $2 \times$ quickly with PBS-T, incubated with DAPI in PBS-T for 5 min at RT, washed $2 \times 5$ min with PBS, and mounted with ProLong Diamond mounting media (Life Technologies). Number of insulator foci per nucleus was 
counted manually. Number of insulator bodies per nucleus and all area measurements of insulator bodies are available in Source Data 7.

Co-immunoprecipitation. Embryonic nuclear extract was prepared from $20 \mathrm{~g}$ of mixed stage (0-24 h) Drosophila embryos ${ }^{50}$. Frozen embryos were ground in liquid nitrogen with mortar and pestle. The following buffers were prepared, and Roche cOmplete protease inhibitor and $1 \mathrm{mM}$ PMSF were added before use: $4 \mathrm{x}$ NB buffer $\left(240 \mathrm{mM} \mathrm{KCl}, 60 \mathrm{mM} \mathrm{NaCl}, 60 \mathrm{mM}\right.$ Tris- $\mathrm{HCl}$ pH 7.4, $\left.20 \mathrm{mM} \mathrm{MgCl}_{2}\right), \mathrm{NB}-1 \mathrm{M}(1 \mathrm{x}$ NB, 0.1 mM EGTA pH 8.0, 0.5 mM DTT, $1 \mathrm{M}$ sucrose), NB*-1M (1 x NB, $0.5 \mathrm{mM}$ DTT, $1 \mathrm{M}$ sucrose), and NB*-1.7M (1 x NB, $0.5 \mathrm{mM}$ DTT, $1.7 \mathrm{M}$ sucrose). Embryo powder was homogenized with $50 \mathrm{~mL}$ NB-1M buffer using glass Kontes dounce tissue grinder and pestle $\mathrm{A}$, and homogenate was cleared by passing through two layered Miracloth at $4{ }^{\circ} \mathrm{C}$. Supernatant was collected by centrifugation at $480 \times g$ for $10 \mathrm{~min}$ at $4^{\circ} \mathrm{C}$, and the pellet was collected by centrifugation at $4300 \times g$ for $10 \mathrm{~min}$ at $4^{\circ} \mathrm{C}$. Then, $20 \mathrm{~mL}$ of $\mathrm{NB}^{*}-1.7 \mathrm{M}$ buffer was overlayed with $20 \mathrm{~mL}$ resuspended pellets in $\mathrm{NB}^{*}-1 \mathrm{M}$ buffer with slight mixing, split into two tubes. Pellets were collected by centrifugation at $19,600 \times g$ for $30 \mathrm{~min}$ at $4{ }^{\circ} \mathrm{C}$. Nuclei were lysed with $5 \mathrm{~mL}$ nuclear lysis buffer HBSMT (50 mM HEPES, pH 6.7; $150 \mathrm{mM}$ $\mathrm{NaCl} ; 5 \mathrm{mM} \mathrm{KCl} ; 2.5 \mathrm{mM} \mathrm{MgCl} 2 ; 0.3 \%$ Triton X-100) supplemented with complete protease inhibitors and $1 \mathrm{mM}$ PMSF and sonicated for 8 cycles with $10 \mathrm{~s}$ on and $50 \mathrm{~s}$ off ${ }^{16}$. The soluble fraction of extracts was collected by centrifugation. First, two sets of $20 \mu \mathrm{L}$ of Protein A or Protein G Sepharose beads (GE Healthcare) were washed three times with nuclear lysis buffer for immunoprecipitation (IP) with antibody raised in guinea pig or in rabbit respectively. Then, $3 \mu \mathrm{L}$ of guinea pig normal serum (Covance Research Products), anti-serum against CP190 (guinea pig), anti-serum against $\mathrm{Su}(\mathrm{Hw})$ (guinea pig), rabbit normal serum (Covance Research Products), anti-serum against Mod(mdg4)67.2 (rabbit), or anti-serum against M1BP (rabbit) was incubated with sepharose beads for $1 \mathrm{~h}$ at $4{ }^{\circ} \mathrm{C}$, and unbound antibodies were removed by centrifugation at $1500 \mathrm{~g}$ for $1 \mathrm{~min}$. Beads were washed three times with $0.2 \mathrm{M}$ of sodium borate, $\mathrm{pH} 9$, and then crosslinked with $20 \mathrm{mM}$ DMP in sodium borate for $30 \mathrm{~min}$ at $\mathrm{RT}^{51}$. Next, beads were collected by centrifugation and washed once with ethanolamine and three times with lysis buffer. After crosslinking, $500 \mu \mathrm{g}$ of nuclear extract was used for each IP and incubated with antibody-bound beads overnight at $4{ }^{\circ} \mathrm{C}$. The next day, beads were collected by centrifugation and washed two times with nuclear lysis buffer HBSMT and once with HBSM (50 mM HEPES, $150 \mathrm{mM} \mathrm{NaCl}, 5 \mathrm{mM} \mathrm{KCl}, 2.5 \mathrm{mM} \mathrm{MgCl} 2$ ). Samples were eluted with SDS sample buffer by boiling, separated using SDSPAGE, transferred to nitrocellulose membrane in $10 \mathrm{mM}$ CAPS, $\mathrm{pH} 11$, and detected using western blotting. The co-immunoprecipitation efficiency was calculated for each immunoprecipitated protein based on the percentage of total input protein.

IP and mass spectrometry. Nuclear extracts from $18 \mathrm{~g}$ of mixed stage $(0-24 \mathrm{~h})$ Drosophila embryos ${ }^{27}$ were lysed in $5 \mathrm{~mL}$ of HBSMT ( $50 \mathrm{mM}$ HEPES, pH 6.7; $150 \mathrm{mM} \mathrm{NaCl} ; 5 \mathrm{mM} \mathrm{KCl} ; 2.5 \mathrm{mM} \mathrm{MgCl} 2 ; 0.3 \%$ Triton X-100) including $1 \mathrm{mM}$ PMSF, and Complete protease inhibitor cocktail (Roche). IP was performed using methods described above, and beads were washed twice with HBSMT, once with HBSM, and eluted with $1 \%$ sodium dodecanoate. Six replicates for each IP were pooled together for mass spectrometry.

Proteins were analyzed using tandem HPLC-mass spectrometry at the NIDDK Mass Spectrometry Facility. Mass from eluted peptides was queried in the UniProt database, and results were analyzed by MaxQuant v1.6.6.0. Top 50 proteins for each pulldown are shown in Supplementary Tables 1 and 2.

Cell lines. Kc167 cells were grown in CCM3 media (Thermo Scientific HyClone, Logan, UT). Cells were maintained in monolayer at $25^{\circ} \mathrm{C}$.

DsRNA knockdowns. A total of $1 \times 10^{7} \mathrm{Kc167}$ cells were transfected with either $20 \mu \mathrm{g}$ of dsRNA against $M 1 B P^{30}, 12 \mu \mathrm{g}$ of dsRNA against $C p 190^{16,28}, 5 \mu \mathrm{g}$ of dsRNA against $\bmod (\operatorname{mdg} 4)$ (specific to deplete the Mod(mdg4)67.2 isoform), or no RNA (mock) using Amaxa cell line Nucleofector kit V (Lonza) and electroporated using G-30 program. Cells were incubated for 5 days at $25^{\circ} \mathrm{C}$ to obtain efficient depletion of each protein but minimal effects on cell viability and growth. The primers for generating the templates for in vitro synthesis of RNAi are listed in Supplementary Table 6 .

Quantification of cell viability. Cell viability was quantified by Trypan Blue exclusion test. Then, $10 \mu \mathrm{L}$ of cells mixed with equal amount of trypan blue was examined to determine the percentage of cells that have clear cytoplasm (viable cells) versus cells that have blue cytoplasm (nonviable cells). Number of viable cells of mock, M1BP dsRNA, or Cp190 dsRNA treated for each day of knockdown were recorded. Viability graphs were generated from the values of viable cell numbers.

Quantification of cell proliferation and mitotic index. Cells were collected each day of knockdown for mock, M1BP dsRNA, or Cp190 dsRNA treatments. Cells were immunostained with rabbit antibody against Histone $\mathrm{H} 3$ (phospho S10) (Abcam ab5176; 1:5000), mouse anti-Tubulin (Sigma T6074; 1:500), labeled with DAPI stain, and mounted with ProLong Diamond mounting media (Life
Technologies) to assay cell viability and mitotic index. Numbers of mitotic cells were counted manually. Statistical tests were performed using Prism 8 software v8.4.2 by GraphPad.

FISH with Oligopaints. Oligopaints were designed to have $80 \mathrm{bp}$ of homology (probes A-F) or $60 \mathrm{bp}$ of homolog (probes $\mathrm{G}-\mathrm{O}$ ) and an average probe density of 5 and 6.5 probes per $\mathrm{kb}$, respectively, using a modified version of the OligoMiner pipeline $^{52}$. Oligopaints were generated in the lab using the T7 method ${ }^{53}$. Briefly, oligo pools were ordered from Twist Bioscience and were amplified by PCR according to instructions from the manufacturer using primers containing the $\mathrm{F}$ and $\mathrm{R}$ barcode sequences added to oligos during the design process. The T7 sequence was added to the reverse primer (TAATACGACTCACTATAGGG), and secondary oligo binding sites were added to the forward primer. T7-labeled PCR products were then in vitro transcribed using the Hi-Scribe T7 RNA synthesis kit (NEB). RNA was converted to RNA:DNA duplexes by reverse transcription using the Thermo Scientific $c^{\text {TM }}$ Maxima $^{\mathrm{m}} \mathrm{H}$ Minus Reverse Transcriptase. RNA was degraded to leave single-stranded DNA FISH probes using alkaline hydrolysis, and probes were purified using the Zymo-100 DNA Clean and Concentrator Kit. Coordinates for all probes and list of primers can be found in Supplementary Tables 8 and 9, respectively. Chromosome 2L and 2R Oligopaints were a gift from E. Joyce ${ }^{54}$.

For FISH, slides were prepared by dispensing $1.5 \times 10^{5}$ cells onto $0.01 \%$ poly-Llysine coated slides and fixed with $4 \%$ paraformaldehyde for $15 \mathrm{~min}$ at RT, followed by washes two times, each for $5 \mathrm{~min}$ in PBS-T. Slides were then permeabilized with PBS- ${ }^{0.5}(0.5 \%$ Triton $\mathrm{X})$ for $15 \mathrm{~min}$ before storing in $70 \%$ ethanol overnight at $-20^{\circ} \mathrm{C}$. The next day, slides were dehydrated in an ethanol row $(90 \%$ ethanol for $5 \mathrm{~min}$ at $-20^{\circ} \mathrm{C}, 100 \%$ ethanol for $5 \mathrm{~min}$ at $-20^{\circ} \mathrm{C}$ ) before allowing them to dry at RT for $5 \mathrm{~min}$. Next, slides were washed once in $2 \mathrm{x}$ SSCT for $5 \mathrm{~min}$ at RT, once in $2 \mathrm{x}$ SSCT/ $50 \%$ formamide for $5 \mathrm{~min}$ at RT, once in $2 \mathrm{x} \mathrm{SSCT} / 50 \%$ formamide at $92^{\circ} \mathrm{C}$ for $2.5 \mathrm{~min}$, and once in $2 \mathrm{x} \mathrm{SSCT} / 50 \%$ formamide at $60^{\circ} \mathrm{C}$ for $20 \mathrm{~min}$. For hybridization, $50-150 \mathrm{pmol}$ of each probe was used per slide in a final volume of $25 \mu \mathrm{L}$. After the applying of primary Oligopaint probes, slides were covered with a coverslip and sealed. Denaturation was performed at $92^{\circ} \mathrm{C}$ for $2.5 \mathrm{~min}$. Slides were transferred to a $37^{\circ} \mathrm{C}$ humidified chamber and incubated for $16-18 \mathrm{~h}$. Then slides were washed in $2 \mathrm{x}$ SSCT at $60^{\circ} \mathrm{C}$ for $15 \mathrm{~min}, 2 \mathrm{x}$ SSCT at RT for $15 \mathrm{~min}$, and $0.2 \mathrm{x}$ $\mathrm{SSC}$ at RT for $5 \mathrm{~min}$. Fluorophore conjugated secondary probes $(0.4 \mathrm{pmol} / \mu \mathrm{L})$ were added to slides, covered, and sealed. Slides were incubated at $37^{\circ} \mathrm{C}$ for $2 \mathrm{~h}$ in a humidified chamber followed by washes as for the primary probes. All slides were washed with DAPI DNA stain for $5 \mathrm{~min}$, followed by $2 \times 5 \mathrm{~min}$ washes in PBS before mounting in Prolong Diamond (Life Technologies).

Imaging, quantification, and data analysis. Images were captured at RT on a Leica DMi 6000B widefield fluorescence microscope using a 1.4 NA $63 \mathrm{x}$ or 100x oil-immersion objective and Leica DFC9000 sCMOS Monochrome Camera. DAPI, CY3, CY5, and FITC filter cubes were used for image acquisition. Images were acquired using LasX Premium software (Leica Application Suite X 3.6.0.20104) and deconvolved using Huygens Professional software v19.10 (Scientific Volume Imaging). After deconvolution, images were segmented and measured using the TANGO 3D-segmentation plugin (v 0.99) for ImageJ (v2.0.0-rc-69/1.53cas) ${ }^{55}$. All nuclei were segmented using the "Hysteresis" algorithm within TANGO. For CT measurements, CTs were segmented using the "Spot Detector 3D" algorithm within TANGO. Foci were segmented using the "Hysteresis" algorithm. To avoid confounding effects of possible differences based on cell cycle stage, imaged nuclei were sorted into G1 and G2 cell cycle stages based on nuclear volume as measured in TANGO (where G1 nuclei were less than $150 \mu \mathrm{m}^{3}$ and G2 nuclei were between 150 and $\left.300 \mu \mathrm{m}^{3}\right)^{54}$. Only G1 nuclei, which would have recently divided during the time of the experiment, were included for all FISH analyses. Statistical tests were performed using Prism 8 (v8.4.2) software by GraphPad. Foci volume measurements are shown in Supplementary Figs $8 \mathrm{~d}$ and 10. All values from TANGO measurements are available in Source Data 8.

ChIP and ChIP-seq library preparation. A total of $2-3 \times 10^{7}$ cells were fixed by adding $1 \%$ formaldehyde directly to cells in culture medium for $10 \mathrm{~min}$ at RT with gentle agitation. Formaldehyde was quenched with $0.125 \mathrm{M}$ glycine with gentle agitation for $5 \mathrm{~min}$. Then cells were pelleted by centrifugation at $2000 \times g$ and washed twice with ice-cold PBS. The pellet was lysed with $0.8 \mathrm{~mL}$ ice-cold cell lysis buffer ( $5 \mathrm{mM}$ PIPES pH 8, $85 \mathrm{mM} \mathrm{KCl,0.5 \%} \mathrm{NP40,} \mathrm{Roche} \mathrm{cOmplete} \mathrm{protease}$ inhibitor). Nuclear pellet was collected by centrifugation at $2000 \times g$ for $5 \mathrm{~min}$ at $4{ }^{\circ} \mathrm{C}$ and resuspended in $1 \mathrm{~mL}$ of nuclear lysis buffer $(50 \mathrm{mM}$ Tris- $\mathrm{HCl} \mathrm{pH} 8$, 10 mM EDTA, $1 \%$ SDS, Roche cOmplete protease inhibitor). Then nuclear lysate was incubated for $10 \mathrm{~min}$ with rotation at $4^{\circ} \mathrm{C}$. After adding $0.5 \mathrm{~mL}$ of IP dilution buffer (16.7 mM Tris- $\mathrm{HCl}$ pH 8, 1.2 mM EDTA, $167 \mathrm{mM} \mathrm{NaCl}, 1.1 \%$ Triton X-100, $0.01 \%$ SDS, Roche cOmplete protease inhibitor) and $300 \mathrm{mg}$ of acid-washed $212-300 \mu \mathrm{m}$ glass beads (Sigma-Aldrich) to nuclear lysate, chromatin was fragmented using a Bioruptor (Diagenode) using 10 cycles of $30 \mathrm{~s}$ on and $30 \mathrm{~s}$ off, maximum output. Lysate was centrifuged at max speed for $10 \mathrm{~min}$ at $4{ }^{\circ} \mathrm{C}$, and supernatant (chromatin) was saved at $-80^{\circ} \mathrm{C}$. Chromatin was diluted to $1: 5$ with IP dilution buffer and added to $50 \mu \mathrm{L}$ prewashed Protein A Sepharose beads (GE Healthcare) and incubated with $5 \mu \mathrm{L}$ of respective antibody overnight at $4{ }^{\circ} \mathrm{C}$ 
with rotation. The next day, beads were washed with the following wash buffers: $3 \times$ with low-salt wash buffer (20 mM Tris-HCl pH 8, 2 mM EDTA, $150 \mathrm{mM} \mathrm{NaCl}, 1 \%$ Triton X-100, 0.1\% SDS), $3 \times$ with high-salt wash buffer ( $20 \mathrm{mM}$ Tris-HCl $\mathrm{pH} 8$, $2 \mathrm{mM}$ EDTA, $500 \mathrm{mM} \mathrm{NaCl}, 1 \%$ Triton X-100, $0.1 \%$ SDS), and $2 \times$ with $\mathrm{LiCl}$ wash buffer (10 mM Tris-HCl pH 8, 1 mM EDTA, $250 \mathrm{mM} \mathrm{LiCl,} \mathrm{1 \%} \mathrm{NP40,} \mathrm{1 \%} \mathrm{deox-}$ ycholate). Then, ChIP sample was eluted $2 \times$ with $200 \mu \mathrm{L}$ of elution buffer $(0.1 \mathrm{M}$ $\mathrm{NaHCO} 3,1 \% \mathrm{SDS}$ ) for $30 \mathrm{~min}$ at $65^{\circ} \mathrm{C}$ in a thermomixer at $800 \mathrm{rpm}$. Eluates were de-crosslinked with solution $(20 \mu \mathrm{L}$ of $5 \mathrm{M} \mathrm{NaCl}, 8 \mu \mathrm{L}$ of $0.5 \mathrm{M}$ EDTA, $10 \mu \mathrm{L}$ of $1 \mathrm{M}$ Tris- $\mathrm{HCl} \mathrm{pH} 8)$ overnight at $65^{\circ} \mathrm{C}$. The next day, $4 \mu \mathrm{L}$ of proteinase $\mathrm{K}(20 \mathrm{mg} / \mathrm{mL})$ was added and incubated for $2 \mathrm{~h}$ at $50^{\circ} \mathrm{C}$, purified using phenol-chloroform followed by ethanol precipitation with $2 \mu \mathrm{L}$ of Glycoblue (Ambion), 0.1 vol of $3 \mathrm{M}$ $\mathrm{NaOAc}$ pH 5.2, $2.5 \mathrm{vol}$ of ice-cold $100 \%$ ethanol and incubated overnight at $-80^{\circ} \mathrm{C}$. Samples were pelleted by centrifugation at high speed $20 \mathrm{~min}$ at $4{ }^{\circ} \mathrm{C}$. After washing with $70 \%$ ethanol, pellets were air-dried at RT and resuspend in $10 \mu \mathrm{L}$ of nuclease-free water. Details of antibodies can be found in Supplementary Table 7. Libraries were constructed by pooling two IP samples using TruSeq adapters (Illumina) according to the TruSeq Illumina ChIP-seq sample preparation protocol with minor modification: after adaptor ligation and PCR amplification, samples were purified using AMPure XP Bead (sample: beads ratio 1:0.8) according to the manufacturer's protocol. All samples were sequenced with HiSeq2500 (Illumina) using $50 \mathrm{bp}$ single-end sequencing.

ChIP-quantitative PCR. We performed quantitative PCR using ChIP DNA samples of normal serum IP (negative control), M1BP IP, CP190 IP, Su(Hw) IP, and Mod(mdg4)67.2 IP from both mock-treated Kc cells and Kc cells transfected with dsRNA against $M 1 B P$ or $C p 190$. ChIP DNA samples were amplified on an Applied Biosystems real-time PCR machine using site-specific primer sets (Supplementary Table 5) and quantified using SYBR Green (Applied Biosystems) incorporation. Experiments were performed with two independent biological replicates, and each sample was quantified using four technical replicates. $C_{t}$ values are available in Source Data 7.

ATAC-seq library preparation. ATAC-seq was performed following a protocol from the Kaestner Lab (https://www.med.upenn.edu/kaestnerlab/assets/usercontent/documents/ATAC-seq\%20Protocol\%20(Omni)\%20-\%20Kaestner\%20Lab. pdf) with minor modifications. A total of $100,000 \mathrm{Kc}$ cells were washed with $50 \mu \mathrm{L}$ cold 1X PBS. Cell pellet was lysed with $50 \mu \mathrm{L}$ cold lysis buffer $(10 \mathrm{mM}$ Tris- $\mathrm{HCl}$, pH 7.5, $10 \mathrm{mM} \mathrm{NaCl}, 3 \mathrm{mM} \mathrm{MgCl}$, 0.1\% NP40, 0.1\% Tween-20, 0.01\% Digitonin) and incubated for $10 \mathrm{~min}$ on ice. Then $500 \mu \mathrm{L}$ of wash buffer $(10 \mathrm{mM}$ Tris-HCl, $\mathrm{pH}$ $7.5,10 \mathrm{mM} \mathrm{NaCl}, 3 \mathrm{mM} \mathrm{MgCl} 2,0.1 \%$ Tween-20) was added to lysate. Nuclei were then collected by centrifuging at $500 \times \mathrm{g}$ for $10 \mathrm{~min}$ at $4{ }^{\circ} \mathrm{C}$ and nuclei were resuspended in $50 \mu \mathrm{L}$ of transposition reaction mix [ $25 \mu \mathrm{L} 2 \mathrm{X}$ TD buffer (Illumina), $16.5 \mu \mathrm{L}$ PBS, $0.5 \mu \mathrm{L}$ 10\% Tween-20, $0.5 \mu \mathrm{L}, 0.5 \mu \mathrm{L}$ 1\% Digitonin, $2.5 \mathrm{uL}$ Tn5 enzyme (Illumina), $5 \mu \mathrm{L}$ nuclease-free water] and incubated for $45 \mathrm{~min}$ at $37^{\circ} \mathrm{C}$ at $1000 \mathrm{rpm}$ (Eppendorf Thermomixer) for fragmentation. DNA was purified with Qiagen Minelute columns, and libraries were amplified by adding $10 \mu \mathrm{L}$ DNA to $25 \mu \mathrm{L}$ of NEBNext HiFi 2x PCR mix (New England Biolabs) and $2.5 \mu \mathrm{L}$ of $25 \mu \mathrm{M}$ each of Ad1 and Ad2 primers using 11 PCR cycles. Libraries were purified with $1.2 \times$ AMPure XP beads. All samples were sequenced with NextSeq-550 (Illumina) using $50 \mathrm{bp}$ paired-end sequencing.

\section{Nascent EU-RNA labeling and library preparation. Nascent EU RNA-seq} (neuRNA-seq) labeling and capture were done by using Click-iT Nascent RNA Capture Kit (Thermo Fisher Scientific) according to the manufacturer's protocol. Kc cells were incubated with $0.2 \mathrm{mM}$ EU for $1 \mathrm{~h}$ and RNA was extracted with Trizol (Thermo Fisher Scientific). Next, RNA was chemically fragmented for 5 min at $70^{\circ} \mathrm{C}$ with RNA Fragmentation Reagents (Thermo Fisher Scientific), followed by DNase I treatment (Roche). Then RNA was ethanol precipitated after Phenol: Chloroform (Thermo Fisher Scientific) purification. The Click-iT reaction was performed with $0.5 \mathrm{mM}$ biotin azide using $5 \mu \mathrm{g}$ of EU-RNA, and biotinylated RNA was captured with $12 \mu \mathrm{L}$ T1 beads. The nascent EU-RNA was used to generate RNA-seq libraries with Ovation RNA-seq Systems 1-16 for Model Organisms (Nugen). Samples were sequenced on HiSeq2500 (Illumina) using 50 bp single-end sequencing at the NIDDK Genomics Core Facility.

Luciferase reporter assays. A total of $5 \times 10^{6} \mathrm{Kc}$ cells were transfected with dsRNA against $M 1 B P, C p 190$, and $\bmod (\operatorname{mdg} 4)$ as described above. After 2 days of incubation with dsRNA at $25^{\circ} \mathrm{C}$, luciferase reporter construct expressing either wild-type or Motif 1 mutant of pGL3-(RpLP1 [-500 to + 50]) or pGL3-(RpLP30 $[-500$ to +50$])$ was co-transfected with RpL-polIII-Renilla into cells. Cells were incubated for an additional 3 days and then assayed in tandem for firefly and Renilla luciferase activity. Cells were lysed and assayed using the dual-luciferase reporter assay system (Promega) according to the manufacturer's protocol. Luciferase activity values are available in Source Data 7 .

ChIP-seq data analysis. FASTQ files of sequenced single-end $50 \mathrm{bp}$ reads were trimmed using cutadapt v1.8.156 with arguments "--quality-cutoff 20," "-a AGATCGGAAGAGC," "--minimum-length 25," and "--overlap 10." Then trimmed reads were mapped to the Flybase r6-24 dm6 genome assembly with Bowtie2 v2.3. $5^{57}$ with default arguments. Multimapping reads were removed mapped reads using samtools v1. $9^{58}$ view command with the argument $-\mathrm{q} 20$. Duplicates were removed from mapped, uniquely mapping reads with picard MarkDuplicates v2.20.2 (http://broadinstitute.github.io/picard/index.html). MACS2 v2.2.55 (https://github.com/taoliu/MACS) was used to call peaks by providing replicate IPs and inputs as multiple BAMs, effectively calling peaks on pooled/merged samples and using additional arguments "-f BAM," "--gsize $=\mathrm{dm}$," "--mfold 3 100" (the latter to include a larger set of preliminary peaks for fragment size estimation).

Binary heatmaps were generated using pybedtools v0.8.160,61. Since peaks for one protein can potentially overlap multiple peaks for other proteins, the output represents unique genomic regions as determined by bedtools multiinter with the -cluster argument. Therefore, as a result, when considering the multi-way overlap with other proteins, the sum of unique genomic regions for a protein is not guaranteed to sum to the total number of called peaks for that protein. Source of called peaks for all other factors are summarized in Supplementary Table 14. Dm3 assembly is lifted over and converted to dm6 (liftover, https://genome.ucsc.edu/cgibin/hgLiftOver).

Pairwise comparison for co-localization of different factors was performed using the BEDTools v2.27.1 "jaccard" command ${ }^{60,61}$. The heatmap was clustered with the scipy.cluster.hierarchy module, using "euclidean" as the distance metric and "Ward" as the clustering method. As the Jaccard statistic is independent of the order of comparison and is symmetric across the diagonal, only the upper triangle is shown.

FlyBase release 6.24 annotations were used to annotate peaks falling into genomic regions in Fig. 3d as follows. Exons were defined as any exon from any transcript of any gene. Introns were defined as the space between exons derived in a per-transcript manner by using the gffutils v0.10.1 (https://github.com/daler/ gffutils) method FeatureDB.create introns(). Promoters were defined as the TSS of each transcript plus $1500 \mathrm{bp}$ upstream. Intergenic regions were defined as all regions between gene bodies. Shared co-bound peaks were determined by using pybedtools BedTool.intersect with the $\mathrm{v}=$ True or $\mathrm{u}=$ True argument, respectively. Each set of peaks was intersected with the annotations using this hierarchy "promoter>exon>intron>intergenic" where a peak was classified according to the highest priority feature. Here, a peak simultaneously intersecting a promoter of one isoform and an intron of a different isoform would be classified as "promoter." To compare percentages across annotated peaks in different types of peaks (All M1BP, all CP190, shared M1BP and CP190 peaks and all gypsy sites), the number of peaks in each class was divided by the total number of peaks of that type.

Heatmaps were generated by using default deepTools v3.4.1 packages in Galaxy. ChIP reads were normalized to reads of input samples and mapped in a $3 \mathrm{~kb}$ window centered on the TSS containing Motif 1 consensus sequence.

Differential ChIP-seq. To detect differential ChIP-seq binding, we used the Diffbind v2.14.0/R package ${ }^{39}$ using the config object "data.frame(RunParallel=TRUE, DataType=DBA_DATA_FRAME, AnalysisMethod=DBA_EDGER, bCorPlot=FALSE, bUsePval=FALSE, fragmentSize $=300$ )" and otherwise used defaults. Input files consisted of the final peak calls mentioned above and the IP and input BAM files for each replicate as described above with multimappers and duplicates removed. Then the final results were exported with the dba.report function with parameters "th $=1$, bCalled=TRUE, bNormalized=TRUE, bCount$s=$ TRUE" and final differentially gained or lost peaks were those that had a $\log 2$ fold change of $>0$ or $<0$, respectively, and $P$ value $<0.05$ ( $\mathrm{R}$ version 4.0.2).

ATAC-seq data analysis. The ATAC-seq data were processed based on the ATAC-seq Guidelines (https://informatics.fas.harvard.edu/atac-seq-guidelines. $\mathrm{html})$. The raw paired-end fastq files trimmed by $51 \mathrm{bp}$ reads were trimmed using cutadapt v1.8.1 $1^{56}$ with arguments "--quality-cutoff 20," "-a AATGATACGGCGA CCACCGAGATCTACACTCGTCGGCAGCGTCAGATGTG-AAATGATACGG CGACCACCGAGATCTACACTCGTCGGCAGCGTCAGATGTG,” “--minimumlength 18." Then trimmed reads were mapped to the Flybase r6-24 dm6 genome assembly with Bowtie2 v2.3.5 $5^{57}$ with "--very-sensitive -X 2000." chrM reads were removed using samtools v1.958. Duplicates were removed with picard MarkDuplicates v2.20.2 (http://broadinstitute.github.io/picard/index.html). We filtered the unmapped reads, non-primary alignment and kept the proper pair reads and unique mapped reads (-f 2 -q 30) using samtools v1.9. MACS2 v2.2.5 $5^{59}$ (https:// github.com/taoliu/MACS) was used to call peaks for only properly paired alignments with "-f BAMPE," “--gsize=dm," “-q 0.0001." For mock, CP190 knockdown and M1BP knockdown, we merged the peak files from three replicates in each condition.

To detect differential ATAC-seq signals, we first merged the peaks in two conditions (CP190 knockdown vs mock and M1BP knockdown vs mock). For ATAC-seq, we did not set "bamControl" in DiffBind. We used the Diffbind v2.14.0/R package ${ }^{39}$ using the config object "data.frame(RunParallel=TRUE, DataType=DBA_DATA_FRAME, AnalysisMethod=DBA_EDGER, bCorPlot= FALSE, bUsePval=FALSE)." Input files consisted of the merged peak files and the BAM files for each replicate. Final differentially gained or lost peaks were those that had a $\log 2$ fold change of $>0$ or $<0$, respectively, and FDR $<0.05$.

For ATAC-seq peak overlap, we used same method as ChIP-seq to produce binary heatmaps using pybedtools v0.8.160,61. We compared mock ChIP-seq peaks and ATAC-seq peaks. 
We applied "computeMatrix" and "plotProfile" in the deeptools package v3.4.1 to plot the ATAC-seq signals around ChIP-seq M1BP or CP190 peaks, TAD borders $^{33}$, and TSS of differential genes after M1BP or Cp190 knockdown.

FET was performed using number of gene promoters occupied/not occupied by decreased/increased ATAC-seq peaks from the group of upregulated (log2fold $>0$, $\left.P_{\text {adj }}<0.05\right)$, downregulated ( $\log 2$ fold $\left.<0, P_{\text {adj }}<0.05\right)$, and not affected gene promoters, respectively, ascertained using DESeq2.

neuRNA-seq (mapping and read counting). Low-quality bases and adaptors were trimmed from sequence reads using cutadapt $\mathrm{v} 2.7^{56}$ with parameters " $-\mathrm{q} 20$ -minimum-length 25 -a AGATCGGAAGAGCACACGTCTGAACTCCAGTCA.” Resulting reads were mapped to the FlyBase r6-24 reference genome using HISAT2 v2.1. $0^{62}$ with default parameters. Aligned reads were counted using subread featureCounts v1.6. $4^{63}$ with default parameters, except that "-t gene" option was used to quantify the reads on gene feature for EuSeq, and "-s1" option specifically for this sense-stranded library. Differential expression analysis was done with DESeq2 $\mathrm{v} 1.22 .1^{64}$ in $\mathrm{v} 3.5 .1 \mathrm{R}$ version.

neuRNA-seq (differential expression). Counts tables were loaded into DESeq2 v1.22.1 for ${ }^{64}$ neuRNA-seq analysis. Counts tables from independent neuRNA-seq experiments were independently imported and normalized using simple design " $\sim$ treatment" and using otherwise default parameters. Differentially expressed genes were those with $P_{\text {adj }}<0.05$.

Fisher's exact tests. These tests were performed using number of gene promoters occupied/not occupied by M1BP or CP190 or both from the group of upregulated $\left(\log 2\right.$ fold $\left.>0, P_{\text {adj }}<0.05\right)$, downregulated $\left(\log 2\right.$ fold $\left.<0, P_{\text {adj }}<0.05\right)$ and not affected gene promoters, respectively analyzed from the DESeq2. Prior to determining the number of peaks intersecting with gene promoter regions from the selected group, the promoter regions were merged if there were any overlapping promoter regions. For intersections with ChIP peaks, a gene promoter region was defined as $1 \mathrm{~kb}$ upand downstream of TSS. The Flybase r6.24 gene annotation gtf file was used to define gene start sites.

FET was used to test whether the up/downregulated genes show preference on the overlapped peaks. FET is implemented by Python scipy package v1.5.2 (scipy. stats.fisher_exact). FET and odds ratios are based on the $2 \times 2$ contingency table. All genes are used. FET test uses two-tailed test.

\begin{tabular}{llll} 
Genes & Up/Down & Others & Row total \\
Overlap with ChIP peaks & $\mathrm{a}$ & $\mathrm{b}$ & $\mathrm{a}+\mathrm{b}$ \\
No overlap & $\mathrm{c}$ & $\mathrm{d}$ & $\mathrm{c}+\mathrm{d}$ \\
Column total & $\mathrm{a}+\mathrm{c}$ & $\mathrm{b}+\mathrm{d}$ & $\mathrm{a}+\mathrm{b}+\mathrm{c}+\mathrm{d}=\mathrm{n}$ \\
\hline
\end{tabular}

The $P$ value is computed as follows:

$$
\begin{gathered}
P=\frac{(a+b) !(c+d) !(a+c) !(b+d) !}{a ! b ! c ! d ! n !} \\
\text { The odds ratio }=\frac{a / b}{c / d}
\end{gathered}
$$

Feature distance analysis to closest TAD border. Four types of TADs (Active, inactive, HP1, and PcG TADs) were downloaded from http://chorogenome.iefreiburg.mpg.de/ ${ }^{33}$ and lifted over to the $\mathrm{dm} 6$ assembly (liftover, https://genome. ucsc.edu/cgi-bin/hgLiftOver). The distance of the feature (promoter or ATAC-seq peak) to the TAD border was computed for each type of TAD, and then pybedtools packages including sort and closest operations were used to search the nearest TAD for each feature. Next, features inside the TAD were selected and computed for the minimum distance to the TAD border. Seaborn/0.9.0 package (seaborn.kdeplot) was used to calculate the kernel density of distance for up, down, and unchanged features, and cumulative distribution plots were generated based on the kernel density estimation. The matplotlib/3.1.1 package was used to plot the density of gene distance to TAD border as a histogram (matplotlib.pyplot.hist, bin=100).

Mann-Whitney $\boldsymbol{U}$ test. The Mann-Whitney $U$ test ("two-sided", scipy.stats. mannwhitneyu, scipy v1.5.2) was used for each set of changed genes against unchanged genes to test whether the difference of both mean TAD border distances is significant. FDR correction was performed using multiple test correction (Benjamini-Hochberg method, statsmodels.stats.multitest.multipletests, statsmodels v0.12.0).

Motif 1 derivation. Motif 1 analysis was derived from the MEME v3.0 analysis of promoters $^{38}$. Motif scanning was carried out using FIMO v5.0.1 ${ }^{65}$ for all gene promoter regions. The Motif 1 _input was a position probability matrix of Motif 1 . The position probability matrix of Motif 1 G[A/C]TACGGTCACACTG was obtained by transformation of a position weight matrix from a previous study ${ }^{38}$.
The transformation converts the weight matrix to the probability position matrix by using the definition

$$
\ln \left(\frac{N_{i j}+p_{i}}{N+1}\right)-\ln \left(p_{i}\right)=M_{i j} .
$$

Here, $M_{i j}$ is the entry of weight matrix and $p_{i}$ the background probability of nucleotide $i$, and $N$ is the total number of sequences used in motif prediction. Assume $p_{i}$ is equal to 0.25 . Since $N$ is much larger than $p_{i}$, the equation can be rewritten into

$$
\ln \left(\frac{N_{i j}}{N+1}\right)-\ln \left(p_{i}\right)=M_{i j} \text { or } \ln \left(P_{i j}\right)-\ln \left(p_{i}\right)=M_{i j} .
$$

$P_{i j}$ is an entry of position probability matrix ${ }^{66}$. After rounding all entries to the 19th decimal, only the probability of the second position was assigned as 0.5 for nucleotide $\mathrm{A}$ and $\mathrm{C}$, respectively. The remaining positions in the matrix were assigned to 1 for each corresponding nucleotide. Other than that, all entries were assigned to zero. The motif search cutoff used was $P \leq 7.12 \mathrm{e}-5$ and shown in Supplementary Data.

Statistics and reproducibility. All quantifications were conducted unblinded and no statistical method was used to predetermine sample size. Statistical parameters including exact value of " $n$," " $P$ " values, and the types of the statistical tests are reported in the figure legends, and " $n$ " of cells examined for four biological replicates for Fig. 8 are summarized in Supplementary Tables 10-12. Error bars are derived from at least three independent biological replicates and displayed on graphs showing either the mean \pm SEM or mean $\pm S D$, as indicated in the figure legends. Immunostaining experiments were performed three times with similar results. All box and whisker plot parameters are defined in the respective figure legends. ChIP-qPCR was conducted using two biological replicates each measured using four technical replicates. Statistical analysis was carried out using Prism 8 (GraphPad Software). All statistical tests used for sequencing data analysis are explained in detail in the "Methods" section. All western blotting experiments were performed two to three times with similar results. Co-immunoprecipitation experiments were performed two times with similar results. Quantitative mass-spec analysis was performed by pooling together six replicates for each IP.

Reporting summary. Further information on experimental design is available in the Nature Research Reporting Summary linked to this paper.

\section{Data availability}

The accession numbers for the raw data FASTQ files, processed files, and BigWig files for all sequencing data generated in this study and deposited in NCBI GEO are GSE142533 and GSE169105. All other publicly available sequencing data analyzed in this study include Kc167 CLAMP GSM2775116, Kc167 BEAF-32 GSM762845, S2 ZIPIC GSM1313421, S2 Pita GSM1313420, S2 Ibf1 GSM1133264, S2 Ibf2 GSM1133265, and Kc167 CTCF GSM1535983. Mass spectrometry data files have been deposited in the PRIDE repository with dataset identifiers PXD026493 for CP190 and PXD026497 for Su $(\mathrm{Hw})$. All other relevant data of this study are available from the corresponding author upon reasonable request. Source Data are provided with this paper.

Received: 28 October 2020; Accepted: 9 June 2021; Published online: 07 July 2021

\section{References}

1. Chen, D. \& Lei, E. P. Function and regulation of chromatin insulators in dynamic genome organization. Curr. Opin. Cell Biol. 58, 61-68 (2019).

2. Rowley, M. J. \& Corces, V. G. Organizational principles of $3 \mathrm{D}$ genome architecture. Nat. Rev. Genet. 19, 789-800 (2018).

3. Kaushal, A. et al. CTCF loss has limited effects on global genome architecture in Drosophila despite critical regulatory functions. Nat. Commun. 12, 1011 (2021)

4. Rowley, M. J. et al. Evolutionarily conserved principles predict 3D chromatin organization. Mol. Cell 67, 837-852 (2017). e837.

5. Hug, C. B., Grimaldi, A. G., Kruse, K. \& Vaquerizas, J. M. Chromatin architecture emerges during zygotic genome activation independent of transcription. Cell 169, 216-228 (2017). e219.

6. Li, L. et al. Widespread rearrangement of $3 \mathrm{D}$ chromatin organization underlies polycomb-mediated stress-induced silencing. Mol. Cell 58, 216-231 (2015).

7. Parkhurst, S. M. et al. The Drosophila su(Hw) gene, which controls the phenotypic effect of the gypsy transposable element, encodes a putative DNAbinding protein. Genes Dev. 2, 1205-1215 (1988).

8. Bonchuk, A., Denisov, S., Georgiev, P. \& Maksimenko, O. Drosophila BTB/ $\mathrm{POZ}$ domains of "ttk group" can form multimers and selectively interact with each other. J. Mol. Biol. 412, 423-436 (2011).

9. Gerasimova, T. I., Gdula, D. A., Gerasimov, D. V., Simonova, O. \& Corces, V. G. A Drosophila protein that imparts directionality on a chromatin insulator is an enhancer of position-effect variegation. Cell 82, 587-597 (1995). 
10. Pai, C. Y., Lei, E. P., Ghosh, D. \& Corces, V. G. The centrosomal protein CP190 is a component of the gypsy chromatin insulator. Mol. Cell 16, 737-748 (2004).

11. Brasset, E. \& Vaury, C. Insulators are fundamental components of the eukaryotic genomes. Heredity (Edinb.) 94, 571-576 (2005).

12. Bushey, A. M., Ramos, E. \& Corces, V. G. Three subclasses of a Drosophila insulator show distinct and cell type-specific genomic distributions. Genes Dev. 23, 1338-1350 (2009).

13. Negre, N. et al. A comprehensive map of insulator elements for the Drosophila genome. PLoS Genet 6, e1000814 (2010).

14. Roseman, R. R., Pirrotta, V. \& Geyer, P. K. The su(Hw) protein insulates expression of the Drosophila melanogaster white gene from chromosomal position-effects. EMBO J. 12, 435-442 (1993).

15. Spana, C., Harrison, D. A. \& Corces, V. G. The Drosophila melanogaster suppressor of Hairy-wing protein binds to specific sequences of the gypsy retrotransposon. Genes Dev. 2, 1414-1423 (1988).

16. Bag, I., Dale, R. K., Palmer, C. \& Lei, E. P. The zinc-finger protein CLAMP promotes gypsy chromatin insulator function in Drosophila. J Cell Sci 132, jcs226092 (2019)

17. Cuartero, S., Fresan, U., Reina, O., Planet, E. \& Espinas, M. L. Ibf1 and Ibf2 are novel CP190-interacting proteins required for insulator function. EMBO J. 33, 637-647 (2014).

18. Maksimenko, O. et al. Two new insulator proteins, Pita and ZIPIC, target CP190 to chromatin. Genome Res. 25, 89-99 (2015).

19. Van Bortle, K. et al. Insulator function and topological domain border strength scale with architectural protein occupancy. Genome Biol. 15, R82 (2014).

20. Schoborg, T., Rickels, R., Barrios, J. \& Labrador, M. Chromatin insulator bodies are nuclear structures that form in response to osmotic stress and cell death. J. Cell Biol. 202, 261-276 (2013).

21. Golovnin, A., Volkov, I. \& Georgiev, P. SUMO conjugation is required for the assembly of Drosophila $\mathrm{Su}(\mathrm{Hw})$ and $\operatorname{Mod}(\operatorname{mdg} 4)$ into insulator bodies that facilitate insulator complex formation. J. Cell Sci. 125, 2064-2074 (2012).

22. Golovnin, A. et al. 'Insulator bodies' are aggregates of proteins but not of insulators. EMBO Rep. 9, 440-445 (2008).

23. Gerasimova, T. I. \& Corces, V. G. Polycomb and trithorax group proteins mediate the function of a chromatin insulator. Cell 92, 511-521 (1998)

24. Matzat, L. H., Dale, R. K., Moshkovich, N. \& Lei, E. P. Tissue-specific regulation of chromatin insulator function. PLoS Genet 8, e1003069 (2012).

25. Matzat, L. H., Dale, R. K. \& Lei, E. P. Messenger RNA is a functional component of a chromatin insulator complex. EMBO Rep. 14, 916-922 (2013).

26. King, M. R., Matzat, L. H., Dale, R. K., Lim, S. J. \& Lei, E. P. The RNA-binding protein Rumpelstiltskin antagonizes gypsy chromatin insulator function in a tissue-specific manner. J. Cell Sci. 127, 2956-2966 (2014).

27. Lei, E. P. \& Corces, V. G. RNA interference machinery influences the nuclear organization of a chromatin insulator. Nat. Genet. 38, 936-941 (2006).

28. Moshkovich, N. et al. RNAi-independent role for Argonaute 2 in CTCF/CP190 chromatin insulator function. Genes Dev. 25, 1686-1701 (2011).

29. Rach, E. A. et al. Transcription initiation patterns indicate divergent strategies for gene regulation at the chromatin level. PLoS Genet 7, e1001274 (2011).

30. Li, J. \& Gilmour, D. S. Distinct mechanisms of transcriptional pausing orchestrated by GAGA factor and M1BP, a novel transcription factor. EMBO J. 32, 1829-1841 (2013).

31. Baumann, D. G. \& Gilmour, D. S. A sequence-specific core promoter-binding transcription factor recruits TRF2 to coordinately transcribe ribosomal protein genes. Nucleic Acids Res 45, 10481-10491 (2017).

32. Cubeñas-Potts, C. et al. Different enhancer classes in Drosophila bind distinct architectural proteins and mediate unique chromatin interactions and $3 \mathrm{D}$ architecture. Nucleic acids Res. 45, 1714-1730 (2017).

33. Ramirez, F. et al. High-resolution TADs reveal DNA sequences underlying genome organization in flies. Nat. Commun. 9, 189 (2018).

34. Sexton, T. et al. Three-dimensional folding and functional organization principles of the Drosophila genome. Cell 148, 458-472 (2012).

35. Hou, C., Li, L., Qin, Z. S. \& Corces, V. G. Gene density, transcription, and insulators contribute to the partition of the Drosophila genome into physical domains. Mol. cell 48, 471-484 (2012).

36. Baumann, D. G. Core Promoter Function in Transcriptional Regulation of Constitutively Expressed Genes in Drosophila. The Pennsylvania State University, 164 (2018).

37. Gdula, D. A., Gerasimova, T. I. \& Corces, V. G. Genetic and molecular analysis of the gypsy chromatin insulator of Drosophila. Proc. Natl Acad. Sci. USA 93, 9378-9383 (1996).

38. Ohler, U., Liao, G.-c., Niemann, H. \& Rubin, G. M. Computational analysis of core promoters in the Drosophila genome. Genome Biol. 3, RESEARCH0087 (2002).

39. Ross-Innes, C. S. et al. Differential oestrogen receptor binding is associated with clinical outcome in breast cancer. Nature 481, 389-393 (2012).
40. Fujioka, M., Mistry, H., Schedl, P. \& Jaynes, J. B. Determinants of chromosome architecture: insulator pairing in cis and in trans. PLOS Genet. 12, e1005889 (2016).

41. Schwartz, Y. B. et al. Nature and function of insulator protein binding sites in the Drosophila genome. Genome Res. 22, 2188-2198 (2012).

42. Mohan, M. et al. The Drosophila insulator proteins CTCF and CP190 link enhancer blocking to body patterning. EMBO J. 26, 4203-4214 (2007).

43. Schwartz, Y. B. et al. Nature and function of insulator protein binding sites in the Drosophila genome. Genome Res 22, 2188-2198 (2012).

44. Shrestha, S., Oh, D. H., McKowen, J. K., Dassanayake, M. \& Hart, C. M. 4Cseq characterization of Drosophila BEAF binding regions provides evidence for highly variable long-distance interactions between active chromatin. PLoS One 13, e0203843 (2018).

45. Kaye, E. G. et al. Differential occupancy of two GA-binding proteins promotes targeting of the Drosophila dosage compensation complex to the male $\mathrm{X}$ chromosome. Cell Rep. 22, 3227-3239 (2018).

46. Bartkuhn, M. et al. Active promoters and insulators are marked by the centrosomal protein 190. EMBO J. 28, 877-888 (2009).

47. Chopra, V. S., Cande, J., Hong, J.-W. \& Levine, M. Stalled Hox promoters as chromosomal boundaries. Genes Dev. 23, 1505-1509 (2009).

48. Zouaz, A. et al. The Hox proteins Ubx and AbdA collaborate with the transcription pausing factor M1BP to regulate gene transcription. EMBO J. 36, 2887-2906 (2017)

49. Markstein, M., Pitsouli, C., Villalta, C., Celniker, S. E. \& Perrimon, N. Exploiting position effects and the gypsy retrovirus insulator to engineer precisely expressed transgenes. Nat. Genet 40, 476-483 (2008).

50. Caravaca, J. M. \& Lei, E. P. Maintenance of a Drosophila melanogaster population cage. J. Vis. Exp. 15, 53756 (2016)

51. Harlow, E. Antibodies: A Laboratory Manual. (Cold Spring Harbor Laboratory Press, New York).

52. Beliveau, B. J. et al. OligoMiner provides a rapid, flexible environment for the design of genome-scale oligonucleotide in situ hybridization probes. Proc. Natl Acad. Sci. 115, E2183 (2018).

53. Nguyen, S. C. \& Joyce, E. F. Programmable chromosome painting with Oligopaints. Methods Mol. Biol. 2038, 167-180 (2019).

54. Rosin, L. F., Nguyen, S. C. \& Joyce, E. F. Condensin II drives large-scale folding and spatial partitioning of interphase chromosomes in Drosophila nuclei. PLoS Genet 14, e1007393 (2018).

55. Ollion, J., Cochennec, J., Loll, F., Escudé, C. \& Boudier, T. TANGO: a generic tool for high-throughput 3D image analysis for studying nuclear organization. Bioinformatics 29, 1840-1841 (2013).

56. Martin, M. Cutadapt removes adapter sequences from high-throughput sequencing reads. EMBnet. Journal 17, 10-12 (2011).

57. Langmead, B. \& Salzberg, S. L. Fast gapped-read alignment with Bowtie 2. Nat. Methods 9, 357-359 (2012)

58. Li, H. et al. The sequence alignment/map format and SAMtools. Bioinformatics 25, 2078-2079 (2009).

59. Zhang, Y. et al. Model-based analysis of ChIP-Seq (MACS). Genome Biol. 9, R137 (2008)

60. Dale, R. K., Pedersen, B. S. \& Quinlan, A. R. Pybedtools: a flexible Python library for manipulating genomic datasets and annotations. Bioinformatics 27, 3423-3424 (2011).

61. Quinlan, A. R. \& Hall, I. M. BEDTools: a flexible suite of utilities for comparing genomic features. Bioinformatics 26, 841-842 (2010).

62. Kim, D., Paggi, J. M., Park, C., Bennett, C. \& Salzberg, S. L. Graph-based genome alignment and genotyping with HISAT2 and HISAT-genotype. Nat. Biotechnol. 37, 907-915 (2019).

63. Liao, Y., Smyth, G. K. \& Shi, W. featureCounts: an efficient general purpose program for assigning sequence reads to genomic features. Bioinformatics $\mathbf{3 0}$, 923-930 (2013).

64. Love, M. I., Huber, W. \& Anders, S. Moderated estimation of fold change and dispersion for RNA-seq data with DESeq2. Genome Biol. 15, 550 (2014).

65. Grant, C. E., Bailey, T. L. \& Noble, W. S. FIMO: scanning for occurrences of a given motif. Bioinformatics 27, 1017-1018 (2011).

66. Hertz, G. Z. \& Stormo, G. D. Identifying DNA and protein patterns with statistically significant alignments of multiple sequences. Bioinformatics 15, 563-577 (1999).

\section{Acknowledgements}

We thank D. Gilmour for sharing previously unpublished results, rabbit anti-M1BP sera, and for comments on the manuscript. We also thank R. Dale for initial software development, A. Beyer for anti-Pep, P. O'Farrell for anti-Pc antibodies, S. Nguyen and E. Joyce for whole chromosome Oligopaints, and J. Kassis, S. Nguyen, and members of the Lei Laboratory for critical reading of the manuscript. This work was funded by the Intramural Program of the National Institute of Diabetes and Digestive and Kidney 
Diseases, National Institutes of Health (DK015602 to E. P. L.). The funders had no role in study design, data collection and analysis, decision to publish, or preparation of the manuscript.

\section{Author contributions}

Conceptualization: I. B., E. P. L.; methodology: I. B.; mass-spec: S. C.; Oligopaints: L. F. R.; software: Y. C., C.-Y. L., G.-Y. Y.; bioinformatics analysis: I. B., S. C.; validation: I. B., E. P. L.; formal analysis: I. B., E. P. L.; investigation: I. B.; data curation: C.-Y. L.; writing - original draft: I. B., E. P. L.; supervision: E. P. L.; funding acquisition: E. P. L.

\section{Funding}

Open Access funding provided by the National Institutes of Health (NIH).

\section{Competing interests}

The authors declare no competing interests.

\section{Additional information}

Supplementary information The online version contains supplementary material available at https://doi.org/10.1038/s41467-021-24407-y.

Correspondence and requests for materials should be addressed to E.P.L.

Peer review information Nature Communications thanks Sergey Razin and the anonymous reviewers for their contribution to the peer review of this work. Peer reviewer reports are available.

Reprints and permission information is available at http://www.nature.com/reprints

Publisher's note Springer Nature remains neutral with regard to jurisdictional claims in published maps and institutional affiliations.

(c) (i) Open Access This article is licensed under a Creative Commons Attribution 4.0 International License, which permits use, sharing, adaptation, distribution and reproduction in any medium or format, as long as you give appropriate credit to the original author(s) and the source, provide a link to the Creative Commons license, and indicate if changes were made. The images or other third party material in this article are included in the article's Creative Commons license, unless indicated otherwise in a credit line to the material. If material is not included in the article's Creative Commons license and your intended use is not permitted by statutory regulation or exceeds the permitted use, you will need to obtain permission directly from the copyright holder. To view a copy of this license, visit http://creativecommons.org/ licenses/by/4.0/.

This is a U.S. Government work and not under copyright protection in the US; foreign copyright protection may apply 2021 